\title{
Estratégias para a atuação pré-hospitalar diante de pacientes com doença avançada em final de vida e suas famílias: revisão integrativa
}

\author{
Strategies for prehospital performance before patients with advanced disease at end-of-life and \\ their families: an integrative review
}

Estrategias para la actuación prehospitalaria ante pacientes con enfermedad avanzada em final de

vida y sus familias: una revisión integradora

\section{Resumo}

Revisão integrativa de literatura que tem por objetivo identificar as estratégias desenvolvidas internacionalmente para a atuação dos profissionais da atenção pré-hospitalar (APH) diante de pacientes com doença avançada em final de vida e suas famílias. As bases de dados consultadas, entre Setembro e Novembro de 2020, foram Scopus, Embase e Web of Science. O material empírico foi composto por 35 artigos, dos quais foram extraídas informações do método e dos resultados em formulário do Google, organizados em planilha no programa Excel e analisados por aproximação temática. Constatou-se que famílias e cuidadores de instituições de longa permanência apresentam medos e anseios diante da dor, da dispneia e da parada cardiorrespiratória, resultando no chamado dos serviços da APH. Os profissionais da $\mathrm{APH}$, ao se depararem com a situação, possuem receios em suspender intervenções e limitações quanto à formação para os cuidados em final de vida. Dessa forma, realizam procedimentos e tendem a encaminhar os pacientes para o hospital, local em que esses acabam morrendo. Sugere-se, em relação aos cuidados com a família, o suporte diante da finitude, por meio da articulação entre os serviços que integram a rede de urgência e emergência, os quais podem favorecer o acompanhamento por serviços da atenção domiciliar ou da própria atenção primária em saúde, visando a permanência no domicílio até a morte. Ademais, demonstra-se relevante o investimento em tecnologias de informação para o compartilhamento de dados clínicos que facilitem a tomada de decisão, bem como a aproximação com serviços especializados em cuidados paliativos.

Palavras-chave: Revisão; Evolução fatal; Serviços médicos de emergência; Assistência pré-hospitalar; Cuidados paliativos.

\footnotetext{
Abstract

Integrative literature review that aimed to identify the strategies internationally developed for the performance of prehospital care professionals before patients with advanced disease at the end-of-life and their families. The databases consulted, between September and November 2020, were Scopus, Embase and Web of Science (WoS). The empirical analysis material was compo sed of 35 articles, from which method information and results were extracted using a Google form, organized in a spreadsheet in the Excel program and analyzed by thematic approach. Three categories were constructed, which indicated that families and caregivers of long-stay institutions present fears and anxieties towards pain, dyspnea and cardiac arrest. This fact results in the call of prehospital care services. Professionals, when come across the situation, are afraid to suspend interventions and have limitations in terms of training for end-of-life care. This way, they perform procedures and tend to lead patients to the hospital, where they die. It is suggested, concerning the care with the family, support towards finitude, through the articulation between the services that are
} 
part of the urgency and emergency network, which can favor monitoring by home care services or primary health care itself, aiming the permanence at home until death. Furthermore, the investment in information technologies for the sharing of clinical data that facilitate decision-making is shown to be relevant, as well as the approximation with palliative care specialized services.

Keywords: Review; Fatal evolution; Emergency medical services; Prehospital care; Palliative care.

\section{Resumen}

Esta revisión integradora objetivó identificar las estrategias desarrolladas internacionalmente para la actuación de los profesionales de la atención prehospitalaria (APH) ante pacientes con enfermedad avanzada en final de vida y sus familias. Las bases de datos consultadas, entre septiembre y noviembre de 2020, fueron Scopus, Embase y Web of Science. El material empírico fue compuesto por 35 artículos, de los cuales fueron extraídas informaciones del método y de los resultados en formulario de Google, organizados en el programa Excel y analizados por aproximación temática. Se constató que familias y cuidadores de asilo de ancianos presentan miedos ante el dolor, la disnea y la parada cardiorrespiratoria, resultando en el llamado de los servicios de APH. Los profesionales de APH tienen miedo de suspender intervenciones y tienen limitaciones en cuanto a la formación para los cuidados al final de vida. Realizan procedimientos y tienden a encaminar a los pacientes hacia el hospital, lugar en el que éstos acaban muriendo. Se sugiere, en relación con los cuidados con la familia, el apoyo ante la finitud, por medio de la articulación entre los servicios que integran la red de urgencia y emergencia, los cuales pueden favorecer el acompañamiento por servicios de la atención domiciliaria o de la propia atención primaria en salud, para permanecer en el domicilio hasta la muerte. Además, se demuestra relevante la inversión en tecnologías de la información para el intercambio de datos clínicos que faciliten la toma de decisiones, así como la aproximación con servicios especializados en cuidados paliativos.

Palabras clave: Revisión. Resultado fatal; Servicios médicos de urgencia; Atención prehospitalaria; Cuidados paliativos.

\section{Introdução}

No ano de 2003, inspirada nos sistemas norte-americano e francês, a Atenção Pré-hospitalar (APH) no Brasil sofreu reformulações com a implantação da Política Nacional de Atenção às Urgências (Brasil, 2003). Para operacionalizar o atendimento mais rápido às situações de urgência e emergência, criou-se o Serviço de Atendimento Móvel de Urgência (SAMU), assistido por um sistema de regulação médica que mantém o contato inicial com a equipe de socorristas (Maier et al., 2016).

Por meio de ligação telefônica para o número 192, o sistema de regulação médica recebe o primeiro contato da ocorrência e, conforme necessidade, comunica e orienta o deslocamento de unidade móvel, com ou sem a presença do médico, para prestação de socorro e atendimento, seja esse em via pública, locais comerciais ou residenciais, até o transporte definitivo para um serviço de atendimento intra-hospitalar (O’Dwyer et al., 2016).

O SAMU é caracterizado pela assistência precoce à vítima que sofre agravo à saúde e que apresenta risco de morte, de forma rápida e com transporte adequado. Embora o foco do SAMU seja em condições agudas, ele também é solicitado quando pessoas com condições crônicas, inclusive em fase avançada, apresentam agudização de algum sintoma (Brasil, 2013).

Doenças crônicas, transmissíveis e não transmissíveis, podem resultar na não responsividade ao tratamento modificador. Quando a doença progride, ou seja, deixa de ser localizada, há comprometimento de órgãos ou estruturas à distância, além da redução da capacidade de funcionamento dos mesmos, e considera-se a doença como em estágio avançado (WHO, [s.d.]; National Cancer Institute, 2011; Ferrell et al., 2017; Tavares et al., 2021). Nesse estágio há um alto risco de óbito e impacto negativo sobre a qualidade de vida devido à sobrecarga de sintomas, ao tratamento e às repercussões da doença na vida do paciente e na dinâmica da família (International Association for Hospice and Palliative Care, 2021).

Por exemplo, a doença renal crônica é considerada avançada quando classificada em estágio quatro ou cinco, momento em que se verifica declínio severo da taxa de filtração glomerular (Tavares et al., 2021; International Association for Hospice and Palliative Care, 2013). Em oncologia, doença avançada é aquela que limita a vida e tem prognóstico estimado entre seis e 24 meses de sobrevida e há presença de metástases à distância (Ferrell et al., 2017). No caso de infecção pelo vírus da Imunodeficiência Humana (HIV), define-se doença avançada quando a contagem de células CD4 é inferior a 200 
células/mm3, estágio três ou quatro em adultos e adolescentes. Nesses casos, como há uma imunodepressão severa e o acometimento por infecções oportunistas se torna frequente, há elevado risco de morte (WHO, [s.d.]). Destaca-se que doença avançada também pode ser identificada, na literatura, como sinônimo de doença terminal ou doença severa/grave (WHO, [s.d.]; International Association for Hospice and Palliative Care, 2021; Ferrell et al., 2017; Tavares et al., 2021).

Para doenças crônicas que não respondem ao tratamento modificador, mas não necessariamente estejam em fase avançada, desde 1990 a Organização Mundial da Saúde (OMS) recomenda o acompanhamento precoce sob a filosofia dos cuidados paliativos (CP). Os CP são uma abordagem multidisciplinar que visa a promoção da qualidade de vida de pessoas com doenças que limitam a continuidade da vida e suas famílias. Por meio de planos de cuidados, os quais contemplam a unidade composta pela pessoa e sua família, objetiva-se a prevenção e o alívio de sintomas, de forma impecável (World Health Organization, 2018).

Recentemente, em 2020, um consenso de especialistas internacionais propôs a ampliação da definição de cuidados paliativos, considerando-os como "cuidados holísticos ativos, ofertados a pessoas de todas as idades que encontram-se em intenso sofrimento relacionados à sua saúde, proveniente de doença severa, especialmente aquelas que estão no final da vida. O objetivo dos Cuidados Paliativos é, portanto, melhorar a qualidade de vida dos pacientes, de suas famílias e de seus cuidadores" (Radbruch et al., 2020, s/p).

Como indicado na atualização do conceito de cuidados paliativos (Radbruch et al., 2020), essa abordagem não é exclusiva ao final da vida, mas pessoas nessa situação e suas famílias se beneficiam ainda mais dela e dos serviços que dela dispõem, devido à onerosidade em termos de sofrimento e estresse que a terminalidade carrega consigo. Assim, estudos (Cordeiro et al., 2020; Hui et al., 2012, 2014) e associações, como a International Association for Hospice and Palliative Care (2016), têm buscado construir uma definição conceitual, que envolve o aspecto temporal, em relação à expressão final da vida. Isso porque o planejamento individual de cuidados deve considerar o grau de dependência e capacidade cognitiva que a pessoa terá em cada etapa da trajetória da doença (Farinholt et al., 2018).

Sabe-se que o momento exato da morte não é possível prever. Entretanto, com base na progressão da doença, no quanto ela afeta a capacidade de realizar atividades básicas da vida diária, o aumento progressivo da dependência, pode-se estimar o período transcorrido entre a definição da doença como avançada e o óbito (Farinholt et al., 2018). Assim, considerase final de vida os últimos 12 meses estimados como tempo de vida (Cordeiro et al., 2020; Hui et al., 2014; International Association for Hospice and Palliative Care, 2016). No final de vida, os dias e horas finais em que ocorre o declínio considerável dos aspectos clínicos e da capacidade do corpo regular a própria existência, são denominados de fase ativa de morte ou fase agônica (Hui et al., 2014; Sobral et al., 2019). É nessa fase que se deve privilegiar aquilo que se nomeia internacionalmente de cuidados terminais, os quais passam a ser centrados exclusivamente no controle de sinais e sintomas que se exacerbam, visando o conforto e a preservação da dignidade (International Association for Hospice and Palliative Care, 2016).

Dentre os sinais e sintomas mais frequentes estão a dor, a dispneia e as intercorrências gastrointestinais (Kobewka et al., 2017). Assim, pode ser difícil e oneroso viabilizar a permanência da pessoa com doença avançada em fase agônica ou em final de vida no domicílio, sobretudo, se não há suporte profissional às famílias ou tecnologias como camas, colchões e medicamentos, que se por um lado podem medicalizar o morrer, por outro podem viabilizar o conforto. Nessas situações, diante do final da vida, especialmente durante a fase ativa de morte, as famílias podem se sentir angustiadas e sem saber como agir. Recorrem, assim, aos serviços de atendimento pré-hospitalar, como o SAMU, para o transporte e encaminhamento do familiar ao hospital (Cordeiro \& Kruse, 2019). Esse encaminhamento, em muitas situações e países, ocorre em virtude da carência de serviços que oferecem cuidados paliativos. 
No mundo, segundo dados da Worldwide Hospice Palliative Care Alliance (WHPCA), em colaboração com a OMS (Worldwide Hospice Palliative Care Alliance, 2020), estima-se que, por ano, 56,8 milhões de pessoas carecem de CP. Dessas, $26 \%$ têm idade entre 20 e 49 anos, $27 \%$ entre 50 e 69 anos e $40 \%$ têm idade igual ou superior a 70 anos. Além disso, $70 \%$ da necessidade de CP são para condições como HIV, doenças cerebrovasculares e demência, apesar do câncer ser o maior grupo único de doenças responsáveis por CP. Como principais barreiras, tem-se a falta de políticas públicas, culturas locais, o pouco conhecimento dos profissionais acerca do $\mathrm{CP}$ e a necessidade de aumentar o número de profissionais para atender a demanda dessas pessoas, o acesso a medicamentos e o desenvolvimento desigual entre os países. Assim, há limitação na oferta dos serviços, com maior acesso por pacientes e famílias que vivem em países de alta renda, sendo que apenas 39\% dos países apresentam disponibilidade de cuidados paliativos na atenção primária.

Em relação à América Latina, entre 17 países que enviaram dados para a Associação Latino-americana de Cuidados Paliativos (ALCP) compor o Atlas sobre CP dessa região (Pastrana et al., 2021), verificou-se aumento, entre 2012 e 2020, no número total de equipes de CP com média de 2,6 equipes/serviços por milhão de habitantes. No entanto, há insuficiência de serviços frente à crescente demanda. Grande parte das equipes (44,8\%) concentra-se na área hospitalar, outra parte $(30,3 \%)$ são equipes mistas, que se dividem entre o hospital e o domicílio, e 24,6\% são exclusivas da atenção primária em saúde. O serviço móvel, caracterizado por equipes denominadas de volantes, interconsultoras ou de consultoria, é o modelo predominante (1,63/milhão de habitantes). Serviços do tipo hospice são os mais escassos (Pastrana et al., 2021). No Brasil, segundo dados da Academia Nacional dos Cuidados Paliativos, existem 191 serviços de cuidados paliativos, dos quais 55\% estão na região Sudeste, concentrados no cenário hospitalar (Santos et al., 2020).

Ao realizar associação livre no motor de busca Google Scholar com os termos "final de vida" e "atendimento préhospitalar", "doença avançada" e "atendimento pré-hospitalar", não foram identificados estudos específicos sobre a temática. Um artigo identificado, referente a um estudo desenvolvido no município de Catanduva/SP, buscou descrever os atendimentos de idosos pelo SAMU entre 2006 e 2012. Dentre os resultados que se aproximam da temática do presente artigo estão que dentre 42.629 pacientes atendidos, 76,5\% foram transferidos para serviços terciários, 23,5\% para unidades básicas de saúde ou de pronto atendimento. Ainda, os autores verificaram que ocorreram 787 óbitos, dos quais 582 foram em domicílio e 205 no momento em que ocorria o deslocamento para o hospital (Gonsaga et al., 2015).

Sendo assim, por não terem sido encontrados estudos específicos, buscou-se alguns que pudessem se aproximar da temática, os quais abordassem cuidados paliativos no cenário de serviços de urgência e emergência intra-hospitalares (Cogo et al., 2020; Formentin et al., 2021; Matos, 2015). Dentre esses, destaca-se o realizado em um serviço de urgência e emergência que identificou alteração de sinais vitais, dor, dispneia, insuficiência respiratória, rebaixamento do sensório e sangramento como os principais motivos de sua procura por pacientes em fim de vida (Formentin et al., 2021). Outro estudo, desenvolvido no mesmo tipo de cenário, revelou falha dos profissionais no cuidado a esses pacientes, além da precária infraestrutura para mantê-los confortáveis, sugerindo medidas institucionais como consolidação de equipes de cuidados paliativos no espaço hospitalar (Matos, 2015). Ao encontro, uma pesquisa constatou que as atitudes e ações de profissionais de saúde de serviços de urgência e emergência são permeadas por desafios em relação aos cuidados paliativos, principalmente quando se trata de proporcionar alívio e conforto ao paciente e aos cuidadores (Cogo et al., 2020).

Evidencia-se, assim, a relevância deste estudo, primeiramente, pela escassez de publicações acerca da temática no cenário brasileiro, o que denota a lacuna na produção do conhecimento. Em termos de impacto social, e como uma segunda justificativa, se reconhece que as equipes de atendimento pré-hospitalar, especialmente com adultos e idosos, são as primeiras a serem acionadas diante de agravos agudos, instabilidades clínicas e para transferências inter-hospitalares (Gonsaga et al., 2015). Dessa forma, essas equipes enfrentam incertezas e dilemas ético-legais sobre as condutas a serem realizadas, notadamente, ao se depararem com pessoas acometidas por doenças crônicas em fase avançada e em fase ativa de morte. Os 
profissionais atuantes em tais equipes possuem formação direcionada ao suporte básico e avançado de vida, os quais pressupõem todos os esforços para trazer e manter a vida daqueles que se encontram sob risco de morte.

Entretanto, nos casos de doença avançada em fase ativa de morte, como já mencionado, tais cuidados não são os recomendados, considerando os princípios da proporcionalidade, do respeito à dignidade e conforto. Dessa forma, as ações e experiências identificadas em estudos internacionais, ao serem aproximadas com a realidade estrutural e organizacional existente no Brasil, podem ser um apontamento na direção em que é possível seguir na elaboração de diretrizes e protocolos de atendimento pelas equipes do SAMU ao se depararem com pessoas com doença avançada e em final de vida, além de suas famílias.

Diante do exposto, delimitou-se como objetivo deste estudo: identificar as estratégias desenvolvidas internacionalmente para a atuação dos profissionais da atenção pré-hospitalar (APH) diante de pacientes com doença avançada em final de vida e suas famílias.

\section{Metodologia}

Revisão integrativa de literatura que cumpriu seis etapas metodológicas: definição do tema e questão de pesquisa, seleção de estudos originais, extração dos dados, avaliação crítica dos estudos, síntese e apresentação dos resultados (Mendes et al., 2019). Destaca-se que, inicialmente, elaborou-se um protocolo de revisão, o qual foi avaliado por duas doutoras na área da Enfermagem. Esse protocolo foi elaborado, sem instrumento específico, mas considerando o referencial metodológico da revisão, pela primeira e segunda autora do artigo, e apresentava justificativa acerca da pesquisa sobre a temática, os objetivos, as bases de dados propostas, descritores a serem associados e suas definições, forma e dados a serem extraídos dos artigos e estratégia para organização e análise dos dados.

Na primeira etapa, utilizando-se da estratégia PICO (Santos et al., 2007) P: paciente - pessoas com doença avançada em final de vida; I: intervenção - estratégias para a atuação dos profissionais da atenção pré-hospitalar em situações de final de vida por doença avançada; C: comparação - não se aplica; Outcomes: resultados - principais efeitos oriundos das estratégias), construiu-se a questão de pesquisa: quais as estratégias desenvolvidas internacionalmente para a atuação dos profissionais da atenção pré-hospitalar diante de pacientes com doença avançada em final de vida e suas famílias?

$\mathrm{Na}$ segunda etapa, como bases de dados para identificação e seleção dos estudos, elegeu-se, pelo rigor nos critérios de indexação de periódicos e documentos, a Scopus e a EMBASE, da editora Elsevier, e a Web of Science (WoS), do grupo Clarivate Analytics, como bases de dados para localização, identificação e seleção dos estudos. $\mathrm{O}$ acesso às bases ocorreu pelo portal de Periódicos da Coordenação de Aperfeiçoamento de Pessoal de Nível Superior (CAPES). Nessa etapa, como critério de qualidade, optou-se pela dupla coleta cega na identificação e seleção dos estudos, a qual foi realizada por duas acadêmicas de enfermagem com experiência em projetos e grupos de pesquisa. Quando houve dúvida quanto à seleção, uma terceira pesquisadora (supervisora da pesquisa) foi consultada para definir o consenso pela exclusão ou inclusão.

Nas bases de dados, associou-se os Medical Subject Headings (Mesh) com o operador booleano AND, conforme apresentado no Quadro 1. 
Quadro 1 - Estratégias de busca.

\begin{tabular}{|c|l|}
\hline Base de dados & \multicolumn{1}{|c|}{ Estratégias de busca } \\
\hline \multirow{3}{*}{ EMBASE } & $\begin{array}{l}\text { \#1Palliative AND care AND emergency AND care, AND prehospital AND \#2 terminal care' } \\
\text { AND emergency AND care, AND prehospital AND \#3 attitude AND to AND death AND } \\
\text { emergency AND care, AND prehospital AND \#4 terminal care' AND emergency AND } \\
\text { medical AND service AND communication AND systems }\end{array}$ \\
\hline \multirow{5}{*}{ Scopus } & (TITLE-ABS-KEY (PALLIATIVE AND CARE) AND TITLE-ABS-KEY (EMERGENCY \\
& AND CARE, AND PREHOSPITAL ) ) OR (TITLE-ABS-KEY (TERMINAL AND CARE) \\
& AND TITLE-ABS-KEY (EMERGENCY AND CARE, AND PREHOSPITAL ) ) AND ( \\
& TITLE-ABS-KEY (ATTITUDE AND TO AND DEATH ) AND TITLE-ABS-KEY \\
& (EMERGENCY AND CARE, AND PREHOSPITAL) AND ( TITLE-ABS-KEY \\
& (TERMINAL AND CARE ) AND TITLE-ABS-KEY (EMERGENCY AND MEDICAL \\
AND SERVICE AND COMMUNICATION AND SYSTEMS) )
\end{tabular}

Fonte: Dados da pesquisa (2020).

Para a seleção dos artigos foram considerados os critérios de inclusão: artigos originais e relatos de experiência, nos idiomas inglês, português, espanhol e francês, disponíveis online na íntegra ou via acesso pelo portal de periódicos CAPES. Foram excluídos artigos de revisão, teses, dissertações, resumos em congresso e artigos de opinião. Não se estabeleceu delimitação temporal. Destaca-se que relatos de experiência foram incluídos, tendo em vista que relatos de estruturação de serviços, desenvolvimentos de ferramentas de comunicação entre os serviços da atenção pré-hospitalar e os demais poderiam ser perdidos, caso esse tipo de artigo não fosse incluído.

Dessa forma, inicialmente, identificou-se 217 artigos na base SCOPUS, 237 na Embase e 69 na WoS. Após a leitura dos títulos, aplicação dos critérios de inclusão e exclusão, leitura dos resumos e estabelecimento do consenso entre as revisoras, 35 artigos integraram a revisão, conforme apresentado na Figura 1.

Figura 1 - Diagrama com a seleção dos estudos.

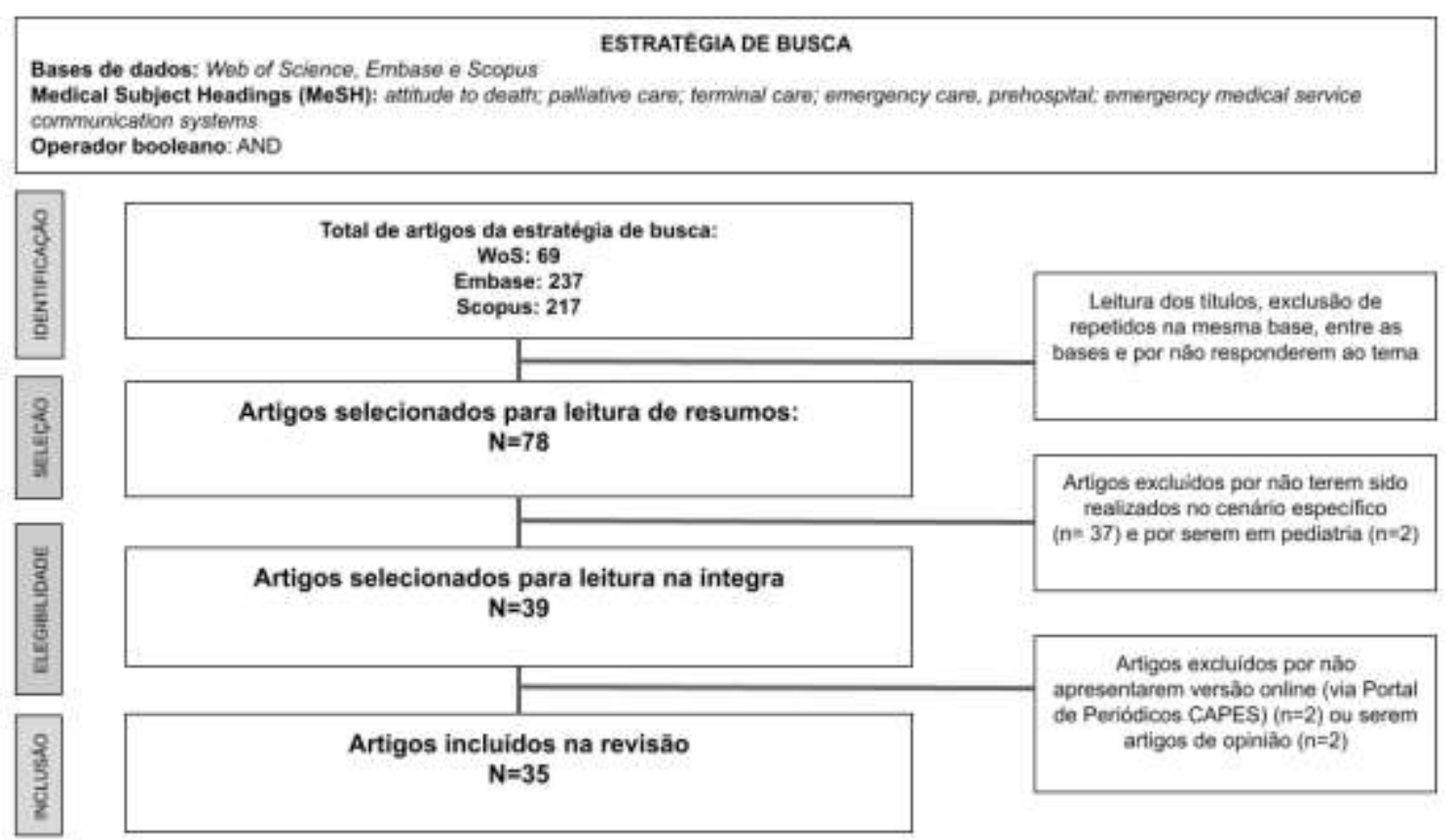

Fonte: Dados da pesquisa (2020). 
Na quarta etapa, recorreu-se ao guia Consolidated Criteria for Reporting Qualitative Research (COREQ) para delimitar as informações a serem extraídas dos estudos, as quais foram preenchidas em formulário do Google. A organização dos dados foi feita em planilha no programa Excel e a análise, quinta etapa da revisão, constituiu-se de duas formas: caracterização dos estudos e formação das unidades temáticas. Essa última foi realizada a partir da similitude entre os achados. Neste estudo, compreende-se o tema como um elemento comum que atravessa um texto, uma fala, uma imagem. Por serem comuns, os temas podem ser agrupados a partir de seus aspectos durante uma investigação, com base na capacidade de análise e olhar direcionado do pesquisador (Polit \& Beck, 2019). A apresentação dos resultados, última etapa da revisão, foi sistematizada de maneira narrativa, sendo que após as análises três unidades temáticas foram construídas: "A iminência da morte e o chamado aos serviços de atendimento pré-hospitalar"; "Desafios da atuação profissional no atendimento préhospitalar em final de vida por doença avançada"; e "Atendimento pré-hospitalar e possíveis estratégias de cuidado diante do final da vida".

\section{Resultados}

Em relação à caracterização, no Quadro 2 apresenta-se a síntese do material empírico de análise.

Quadro 2 - Material empírico de análise.

\begin{tabular}{|c|c|c|c|}
\hline $\begin{array}{l}\text { Código } \\
\text { artigo }\end{array}$ & Título & Autores & Ano \\
\hline A1 & $\begin{array}{l}\text { Response of paramedics to terminally ill patients with } \\
\text { cardiac arrest: an ethical dilemma }\end{array}$ & $\begin{array}{l}\text { Guru, V.; Verbeek, P.R.; } \\
\text { Morrison, L.J. }\end{array}$ & 1999 \\
\hline A2 & $\begin{array}{c}\text { Prehospital withholding and withdrawal of life-sustaining } \\
\text { treatments. The French LATASAMU survey }\end{array}$ & Ferrand, E., Marty, J. & 2006 \\
\hline A3 & $\begin{array}{c}\text { Withholding resuscitation: A new approach to prehospital } \\
\text { end-of-life decisions }\end{array}$ & $\begin{array}{l}\text { Feder, S., Matheny, R.L., } \\
\text { Loveless Jr., R.S., Rea, T.D. }\end{array}$ & 2006 \\
\hline A4 & $\begin{array}{c}\text { Emergency calls and need for emergency care in patients } \\
\text { looked after by a palliative care team: retrospective } \\
\text { interview study with bereaved relatives }\end{array}$ & $\begin{array}{c}\text { Wiese, C.H.R., Vossen- } \\
\text { Wellmann, A., Morgenthal, } \\
\text { H.C., Popov, A.F., Graf, B.M., } \\
\text { Hanekop, G.G. } \\
\end{array}$ & 2008 \\
\hline A5 & $\begin{array}{c}\text { The law number 2005-370 of April 22, } 2005 \text { concerning } \\
\text { the patients' rights at the end of life: a case of prehospital } \\
\text { medicine }\end{array}$ & $\begin{array}{l}\text { Jabre, P., Combes, X., Marty, J., } \\
\text { Margenet, A., Ferrand, E. }\end{array}$ & 2008 \\
\hline A6 & $\begin{array}{l}\text { Paramedic knowledge, attitudes, and training in end-of-life } \\
\text { care }\end{array}$ & $\begin{array}{c}\text { Stone, S.C., Abbott, J., } \\
\text { McClung, C.D., Colwell, C.B., } \\
\text { Eckstein, M., Lowenstein, S.R. }\end{array}$ & 2009 \\
\hline A7 & $\begin{array}{l}\text { Quality of out-of-hospital palliative emergency care } \\
\text { depends on the expertise of the emergency medical team-a } \\
\text { prospective multi-centre analysis }\end{array}$ & $\begin{array}{l}\text { Wiese C.H.R., Bartels U.E., } \\
\text { Marczynska K., Ruppert D., } \\
\text { Graf B.M., Hanekop G.G. } \\
\end{array}$ & 2009 \\
\hline A8 & $\begin{array}{l}\text { Treatment of palliative care emergencies by prehospital } \\
\text { emergency physicians in Germany: an interview based } \\
\text { investigation }\end{array}$ & $\begin{array}{l}\text { Wiese, C.H.R.; Bartels, U.E.; } \\
\text { Ruppert, D.; Marung, H.; Luiz, } \\
\text { T.; Graf, B.M.; Hanekop, G.G. }\end{array}$ & 2009 \\
\hline A9 & $\begin{array}{l}\text { Prehospital emergency treatment of palliative care patients } \\
\text { with cardiac arrest: a retrolective investigation }\end{array}$ & $\begin{array}{l}\text { Wiese, C.H.R.; Bartels, U.E.; } \\
\text { Zausig, Y.A.; Pfirstinger, J.; } \\
\text { Graf, B.M.; Hanekop, G.G. } \\
\end{array}$ & 2010 \\
\hline A10 & $\begin{array}{c}\text { Prehospital emergency physicians' experiences with } \\
\text { advance directives in Germany: a questionnaire-based } \\
\text { multicenter study }\end{array}$ & $\begin{array}{l}\text { Wiese, C.H.R.; Bartels, U.E.; } \\
\text { Ruppert, D.B.; Graf, B.M.; } \\
\text { Hanekop, G.G. } \\
\end{array}$ & 2011 \\
\hline A11 & $\begin{array}{c}\text { Collaboration between prehospital emergency medical } \\
\text { teams and palliative care networks allows a better respect } \\
\text { of a patient's will }\end{array}$ & $\begin{array}{l}\text { Burnod, A.; Lenclud, G.; Ricard- } \\
\text { Hibon, A.; Juvin, P.; Mantz, J.; } \\
\text { Duchateau, F.X. } \\
\end{array}$ & 2012 \\
\hline A12 & $\begin{array}{l}\text { Paramedics experiences and expectations concerning } \\
\text { advance directives: a prospective, questionnaire-based, bi- } \\
\text { centre study }\end{array}$ & $\begin{array}{l}\text { Taghavi, M.; Simon, A.; } \\
\text { Kappus, S.; Meyer, N.; Lassen, } \\
\text { C.L.; Klier, T.; Ruppert, D.B.; } \\
\text { Graf, B.M.; Hanekop, G.G.; } \\
\text { Wiese, C.H.R. }\end{array}$ & 2012 \\
\hline
\end{tabular}




\begin{tabular}{|c|c|c|c|}
\hline A13 & $\begin{array}{l}\text { Paramedics' perceptions of their role in palliative care: } \\
\text { analysis of focus group transcripts }\end{array}$ & $\begin{array}{l}\text { Lord, B., Récoché, K., } \\
\text { O'Connor, M., Yates, P., } \\
\text { Service, M. } \\
\end{array}$ & 2012 \\
\hline A14 & $\begin{array}{l}\text { International recommendations for outpatient palliative } \\
\text { care and prehospital palliative emergencies: a prospective } \\
\text { questionnaire-based investigation }\end{array}$ & $\begin{array}{l}\text { Wiese, C.H.R.; Lassen, C.L.; } \\
\text { Bartels, U.E.; Taghavi, M.; } \\
\text { Elhabash, S.; Graf, B.M.; } \\
\text { Hanekop, G.G. } \\
\end{array}$ & 2013 \\
\hline A15 & $\begin{array}{c}\text { Breaking down the silos: collaboration delivering an } \\
\text { efficient and effective response to palliative care } \\
\text { emergencies }\end{array}$ & $\begin{array}{l}\text { Swetenham, K., Grantham, H., } \\
\text { Glaetzer, K. }\end{array}$ & 2014 \\
\hline A16 & $\begin{array}{c}\text { Palliative Care and Prehospital Emergency Medicine } \\
\text { analysis of a case series }\end{array}$ & $\begin{array}{l}\text { Carron, P.N.; Dami, F.; Diawara, } \\
\text { F.; Hurst, S.; Hugli, O. }\end{array}$ & 2014 \\
\hline A17 & $\begin{array}{c}\text { Preparation for frontline end-of-life care: exploring the } \\
\text { perspectives of paramedics and emergency medical } \\
\text { technicians }\end{array}$ & $\begin{array}{l}\text { Waldrop D.P., Clemency B., } \\
\text { Maguin E., Lindstrom H. }\end{array}$ & 2014 \\
\hline A18 & $\begin{array}{l}\text { "We Are Strangers Walking Into Their Life-Changing } \\
\text { Event": how prehospital providers manage emergency } \\
\text { calls at the end of life }\end{array}$ & $\begin{array}{l}\text { Waldrop, D.P.; Clemency, B.; } \\
\text { Lindstrom, H.A.; Cordes, C.C. }\end{array}$ & 2015 \\
\hline A19 & $\begin{array}{l}\text { Paramedics' perceptions and educational needs with } \\
\text { respect to palliative care }\end{array}$ & $\begin{array}{l}\text { Rogers I.R., Shearer F.M., } \\
\text { Rogers J.R., Ross-Adjie G., } \\
\text { Monterosso L., Finn J. } \\
\end{array}$ & 2015 \\
\hline A20 & $\begin{array}{l}\text { Prehospital providers' perceptions of emergency calls near } \\
\text { life's end }\end{array}$ & $\begin{array}{l}\text { Waldrop, D.; Clemency, B.; } \\
\text { Maguin, E.; Lindstrom, H. }\end{array}$ & 2015 \\
\hline A21 & $\begin{array}{c}\text { Exploring New Zealand paramedic attitudes towards } \\
\text { advance directives: an ethical analysis }\end{array}$ & Davey, P.; Lees, A.; Godbold, R. & 2016 \\
\hline A22 & $\begin{array}{l}\text { Paramedics' experiences of end-of-life care decision } \\
\text { making with regard to nursing home residents: an } \\
\text { exploration of influential issues and factors }\end{array}$ & Murphy-Jones, G., Timmons, S. & 2016 \\
\hline $\mathbf{A 2 3}$ & $\begin{array}{c}\text { Retentissement psychologique et connaissances par } \\
\text { rapport à la fin de vie em médecine d'urgence pré- } \\
\text { hospitalière chez les médecins du SMUR - Résultats de } \\
\text { l'ênquete nationale de } 2013\end{array}$ & $\begin{array}{l}\text { Jouffroy R., Michaloux M., } \\
\text { Khelifi G., Guyard A., Philippe } \\
\text { P., Carli P., Vivien B. }\end{array}$ & 2017 \\
\hline A24 & Innovative urgent care for the palliative patient at home & $\begin{array}{l}\text { Montgomery, C.L.; Pooler, C.; } \\
\text { Arsenault, J.E.; Berean, C.; } \\
\text { Sharman, R.; Cameron, C.L.; De } \\
\text { Kock, I. }\end{array}$ & 2017 \\
\hline A25 & $\begin{array}{l}\text { Ambulance staff and end-of-life hospital admissions: a } \\
\text { qualitative interview study }\end{array}$ & $\begin{array}{l}\text { Hoare, S.a; Kelly, M.P.; } \\
\text { Prothero, L.; Barclay, S. }\end{array}$ & 2018 \\
\hline A26 & $\begin{array}{c}\text { Mediating systems of care: emergency calls to long-term } \\
\text { care facilities at life's end }\end{array}$ & $\begin{array}{c}\text { Waldrop D.P., McGinley J.M., } \\
\text { Clemency B. } \\
\end{array}$ & 2018 \\
\hline A27 & $\begin{array}{l}\text { Adherence to "no transfer to hospital" advance directives } \\
\text { among nursing home residents }\end{array}$ & $\begin{array}{c}\text { Nemiroff, L., Marshall, E.G., } \\
\text { Jensen, J.L., Clarke, B., Andrew, } \\
\text { M.K. }\end{array}$ & 2019 \\
\hline A28 & $\begin{array}{c}\text { Retentissement psychologique des Situações de fin de vie } \\
\text { en médecine d'urgence préhospitalière chez les médecins } \\
\text { du Samu de Paris }\end{array}$ & $\begin{array}{c}\text { Jouffroy R., Michaloux M., } \\
\text { Khelifi G., Guyard A., Philippe } \\
\text { P., Carli P., Vivien B. } \\
\end{array}$ & 2017 \\
\hline A29 & $\begin{array}{c}\text { Challenges faced by prehospital emergency physicians } \\
\text { providing emergency care to patients with advanced } \\
\text { incurable diseases }\end{array}$ & $\begin{array}{l}\text { Kamphausen, A.; Roese, H.; } \\
\text { Oechsle, K.; Issleib, M.; Zollner, } \\
\text { C.; Bokemeyer, C.; Ullrich, A. }\end{array}$ & 2019 \\
\hline A30 & $\begin{array}{l}\text { Decision-making in the moments before death: challenges } \\
\text { in prehospital care }\end{array}$ & $\begin{array}{l}\text { Waldrop, D.P., McGinley, J.M., } \\
\text { Dailey, M.W., Clemency, B. }\end{array}$ & 2019 \\
\hline A31 & $\begin{array}{c}\text { Paramedic information needs in end-of-life care: a } \\
\text { qualitative interview study exploring access to a shared } \\
\text { electronic record as a potential solution }\end{array}$ & $\begin{array}{l}\text { Patterson, R., Standing, H., Lee, } \\
\text { M., Dalkin, S., Lhussier, M., } \\
\text { Exley, C., Brittain, K. } \\
\end{array}$ & 2019 \\
\hline A32 & $\begin{array}{l}\text { Paramedics providing palliative care at home: a mixed- } \\
\text { methods exploration of patient and family satisfaction and } \\
\text { paramedic comfort and confidence }\end{array}$ & $\begin{array}{c}\text { Carter, A.J.E., Arab, M., } \\
\text { Harrison, M., Goldstein, J., } \\
\text { Stewart, B., Lecours, M., } \\
\text { Sullivan, J., Villard, C., Crowell, } \\
\text { W., Houde, K., Jensen, J.L., } \\
\text { Downer, K., Pereira, J. } \\
\end{array}$ & 2019 \\
\hline A33 & $\begin{array}{l}\text { Transport home and terminal extubation by emergency } \\
\text { medical services: an example of innovation in end-of-life } \\
\text { care }\end{array}$ & $\begin{array}{l}\text { Clemency, B.M.; Grimm, K.T.; } \\
\text { Lauer, S.L.; Lynch, J.C.; } \\
\text { Pastwik, B.L.; Lindstrom, H.A.; } \\
\text { Dailey, M.W.; Waldrop, D.P. }\end{array}$ & 2019 \\
\hline
\end{tabular}




\begin{tabular}{|c|c|c|c|}
\hline $\mathbf{A 3 4}$ & Managing death in the field: prehospital end-of-life care & $\begin{array}{c}\text { Waldrop D.P., Waldrop M.R., } \\
\text { McGinley J.M., Crowley C.R., } \\
\text { Clemency B. }\end{array}$ & 2020 \\
\hline $\mathbf{A 3 5}$ & $\begin{array}{c}\text { South African paramedic perspectives on prehospital } \\
\text { palliative care }\end{array}$ & $\begin{array}{c}\text { Gage C.H., Geduld H., Stassen } \\
\text { W. }\end{array}$ & 2020 \\
\hline
\end{tabular}

Fonte: Dados da pesquisa (2020).

Os países com mais publicações foram, Estados Unidos da América (Feder et al., 2006; Stone et al., 2009; Waldrop et al., 2015; Waldrop et al., 2014, 2015, 2018, 2019, 2020), Alemanha (Kamphausen et al., 2019; Wiese et al., 2008; Wiese, Bartels, Marczynska, et al., 2009; Wiese, Bartels, Ruppert, et al., 2009; Wiese et al., 2010, 2011) e França (Burnod et al., 2012; Ferrand et al., 2006; Jabre et al., 2008; Jouffroy et al., 2017b, 2017a). Quanto à área de publicação do estudo, 22 artigos foram publicados por profissionais da Medicina (Burnod et al., 2012; Carron et al., 2014; Feder et al., 2006; Ferrand et al., 2006; Gage et al., 2020; Guru et al., 1999; Jabre et al., 2008; Jouffroy et al., 2017b, 2017a; Kamphausen et al., 2019; MurphyJones \& Timmons, 2016; Nemiroff et al., 2019; Stone et al., 2009; Taghavi et al., 2012; Waldrop et al., 2015; Waldrop et al., 2015; Wiese et al., 2008, 2013; Wieseet al., 2009; Wieseet al., 2009; Wiese et al., 2010, 2011), os outros 13 artigos foram multidisciplinares (Carter et al., 2019; Clemency et al., 2019; Davey et al., 2016; Hoare et al., 2018; Lord et al., 2012; Montgomery et al., 2017; Patterson et al., 2019; Rogers et al., 2015; Swetenham et al., 2014; Waldrop et al., 2014, 2018, 2019, 2020). Quanto ao tipo de abordagem utilizada nos estudos, 16 artigos apresentaram abordagem qualitativa (Carron et al., 2014; Clemency et al., 2019; Gage et al., 2020; Hoare et al., 2018; Jabre et al., 2008; Kamphausen et al., 2019; Lord et al., 2012; Montgomery et al., 2017; Murphy-Jones \& Timmons, 2016; Patterson et al., 2019; Waldrop et al., 2015; Waldrop et al., 2015, 2018, 2019; Wiese et al., 2013; Wiese et al., 2010), 10 quanti-qualitativa (Davey et al., 2016; Feder et al., 2006; Ferrand et al., 2006; Guru et al., 1999; Jouffroy et al., 2017b, 2017a; Swetenham et al., 2014; Wiese et al., 2008; Wieseet al., 2009; Wiese et al., 2011), seis métodos mistos (Carter et al., 2019; Nemiroff et al., 2019; Rogers et al., 2015; Taghavi et al., 2012; Waldrop et al., 2014, 2020) e três quantitativa (Burnod et al., 2012; Stone et al., 2009; Wieseet al., 2009).

Sobre os autores, 81 eram do sexo feminino e 108 do sexo masculino. Em relação ao vínculo que eles possuíam com instituições, 31 artigos eram de autores filiados à universidades e 14 também tinham vínculos com hospitais. Em relação ao tipo de documento, 31 artigos eram oriundos de pesquisa original (Burnod et al., 2012; Carter et al., 2019; Davey et al., 2016; Feder et al., 2006; Ferrand et al., 2006; Gage et al., 2020; Guru et al., 1999; Hoare et al., 2018; Jouffroy et al., 2017b, 2017a; Kamphausen et al., 2019; Lord et al., 2012; Murphy-Jones \& Timmons, 2016; Nemiroff et al., 2019; Patterson et al., 2019; Rogers et al., 2015; Stone et al., 2009; Swetenham et al., 2014; Taghavi et al., 2012; Waldrop et al., 2015; Waldrop et al., 2014, 2015, 2018, 2019, 2020; Wiese et al., 2008, 2013; Wieseet al., 2009; Wieseet al., 2009; Wiese et al., 2010, 2011) e quatro eram relatos de experiência (Carron et al., 2014; Clemency et al., 2019; Jabre et al., 2008; Montgomery et al., 2017). Quanto ao(s) cenário(s) dos estudos, 34 foram desenvolvidos em serviço de atendimento móvel de urgência/pré-hospitalar, mas não somente nele (Burnod et al., 2012; Carron et al., 2014; Carter et al., 2019; Davey et al., 2016; Feder et al., 2006; Ferrand et al., 2006; Gage et al., 2020; Guru et al., 1999; Hoare et al., 2018; Jabre et al., 2008; Jouffroy et al., 2017a, 2017b; Kamphausen et al., 2019; Lord et al., 2012; Montgomery et al., 2017; Murphy-Jones \& Timmons, 2016; Nemiroff et al., 2019; Patterson et al., 2019; Rogers et al., 2015; Stone et al., 2009; Swetenham et al., 2014; Taghavi et al., 2012; Waldrop et al., 2015; Waldrop et al., 2014, 2015, 2018, 2019, 2020; Wiese et al., 2008, 2013; C. H. R. Wieseet al., 2009; Wieseet al., 2009). Outros cenários foram o domicílio (Burnod et al., 2012; Carter et al., 2019; Clemency et al., 2019; Davey et al., 2016; Feder et al., 2006; Guru et al., 1999; Jabre et al., 2008; Kamphausen et al., 2019; Montgomery et al., 2017; Swetenham et al., 2014; Wiese et al., 2008; Wiese, Bartels, Ruppert, et al., 2009; Wiese et al., 2010) e instituições de longa permanência de idosos (Montgomery et al., 2017; Murphy-Jones \& Timmons, 2016; Nemiroff et al., 2019; Waldrop et al., 2018; Wieseet al., 2009; Wiese et al., 2010). Todos os estudos desenvolvidos nesses cenários apresentavam a interface com o final da vida ou com os cuidados paliativos, 
entretanto, como já mencionado anteriormente, nenhum deles foi desenvolvido no Brasil. A respeito dos participantes, 21 artigos contemplaram exclusivamente profissionais de saúde (Davey et al., 2016; Ferrand et al., 2006; Gage et al., 2020; Hoare et al., 2018; Jouffroy et al., 2017b, 2017a; Kamphausen et al., 2019; Lord et al., 2012; Murphy-Jones \& Timmons, 2016; Patterson et al., 2019; Rogers et al., 2015; Stone et al., 2009; D. Waldrop et al., 2015; Waldrop et al., 2014, 2015, 2018, 2019, 2020; Wiese et al., 2013; Wieseet al., 2009; Wiese et al., 2011), 13 envolveram pacientes e familiares (Burnod et al., 2012; Carron et al., 2014; Carter et al., 2019; Clemency et al., 2019; Feder et al., 2006; Jabre et al., 2008; Montgomery et al., 2017; Nemiroff et al., 2019; Swetenham et al., 2014; Taghavi et al., 2012; Wiese et al., 2008; Wieseet al., 2009; Wiese et al., 2010) e um não envolveu participantes (Guru et al., 1999).

Após as análises, três unidades temáticas foram construídas: "A iminência da morte e o chamado aos serviços de atendimento pré-hospitalar"; "Desafios da atuação profissional no atendimento pré-hospitalar em final de vida por doença avançada"; e "Atendimento pré-hospitalar e possíveis estratégias de cuidado diante do final da vida".

\section{A iminência da morte e o chamado aos serviços de atendimento pré-hospitalar}

Os motivos que mais levaram ao chamado do serviço de atendimento pré-hospitalar foram parada cardiorrespiratória (Guru et al., 1999; Wiese et al., 2013) ou agudização de sintomas inerentes à fase ativa de morte, especialmente a dispneia e a dor (Montgomery et al., 2017; Waldrop et al., 2020; Wiese et al., 2008), mas também distúrbios neurológicos (Burnod et al., 2012). Ainda, a ausência de suporte por alguma equipe, o medo diante da morte de alguém (Swetenham et al., 2014) ou a sensação de não saber como reagir frente à situação também foram motivos evidenciados (Lord et al., 2012; Montgomery et al., 2017). Tal medo esteve relacionado à sobrecarga do cuidador familiar, pouco preparo para responder a situações de emergência, desamparo (Feder et al., 2006; Kamphausen et al., 2019) e à presença de um familiar distante naquele momento (Wiese et al., 2008).

Equipes de instituições de longa permanência ou clínicas também apresentaram dificuldades diante da morte, acionando serviços de emergência quando os residentes apresentavam declínio clínico. Estresse e ansiedade estavam associados às reações pessoais dos membros da equipe à perda e pelo desejo da morte não ocorrer naquele local (Waldrop et al., 2018). Mesmo em serviços de hospice, em que pacientes recebem cuidados paliativos, houveram ligações aos serviços de atendimento pré-hospitalar, como uma resposta automática à angústia percebida pelos profissionais (Stone et al., 2009; Waldrop et al., 2020).

Assim, constatou-se que quando familiares e/ou cuidadores se deparam com possibilidade real da morte, ocorre o desespero e eles tendem a entrar em conflito com os desejos do próprio paciente. Ao reconhecerem a agudização de sintomas, eles chamam o serviço de APH, divergindo de decisões, inclusive, dos profissionais desse serviço (Ferrand et al., 2006). Em um dos estudos, observou-se que a ressuscitação indesejada tem sido abordada por meio de educação com as famílias, com vistas a evitar ligações ao serviço de atendimento pré-hospitalar, porém, diante da morte, por vezes, é inevitável o chamado (Feder et al., 2006).

Profissionais de um estudo (Waldrop et al., 2015) descreveram ligações em que familiares solicitaram o transporte de pessoas que recusaram a hospitalização, sentindo-se diante de um "sequestro" ao ter que retirar o paciente do domicílio. Normalmente, essas decisões eram tomadas por familiares não envolvidos com os cuidados e decisões e que não tinham conhecimento das vontades da pessoa. Para minimizar essas situações, destacou-se, em outro estudo, a importância de identificar um familiar tomador de decisão, para reduzir os conflitos com as famílias, bem como melhorar o processo de comunicação com as mesmas (Kamphausen et al., 2019).

Diante dos pacientes e familiares, evidenciou-se que a maior parte dos profissionais não se sente confortável em não fazer o transporte hospitalar quando identificam que a morte é iminente. Apesar disso, eles se sentem à vontade para lidar com 
a resposta emocional de uma família à morte, a qual tende a ser intensa frente ao corpo morto no domicílio após tentativas de ressuscitação (Waldrop et al., 2020).

De forma a reduzir desconfortos e dilemas por parte dos profissionais, nos estudos analisados indicou-se como estratégia o uso das manifestações de vontade sobre os desejos em final de vida (Taghavi et al., 2012; Waldrop et al., 2020). Essas manifestações podem ser feitas por documentos ou verbalmente, dependendo da legislação de cada país, e foram apontadas como necessárias por serem o registro formal sobre cuidados ou medidas terapêuticas em final de vida, respaldando o atendimento das equipes de serviço pré-hospitalar diante dos chamados. Tais equipes podem se sentir mais seguras quando encontram manifestações de vontade, especialmente sob a forma de registro escrito. Isso porque ao se depararem com os desejos documentados, sentem-se menos receosos em suspender ou limitar condutas terapêuticas, decisões que em casos em que não há esse tipo de documento podem resultar em impasses éticos e jurídicos com a família.

Dessa forma, na presente revisão, as manifestações de vontade identificadas foram a ordem de não ressuscitar (Do Not Resuscitate - DNR) ou Ordem de não tentar ressuscitar (Do not attempt resuscitation -DNAR), o cartão para crises paliativas (Palliative Crisis Card - PCC) e o documento de Ordens Médicas para Tratamentos que mantém a Vida (Medical Orders for Life-Sustaining Treatment - MOLST/ Physician Orders for Life-Sustaining Treatment - POLST) (Taghavi et al., 2012; D. P. Waldrop et al., 2020). Os pedidos de DNR são mais disseminados e reconhecidos se comparados com documentos recentes como o MOLST (Waldrop et al., 2020).

O cartão para crises paliativas (Palliative Crisis Card - PCC), utilizado em serviços de atendimento pré-hospitalar na Alemanha, por cuidadores e profissionais de saúde, informa a não tentativa de ressuscitação se o paciente for encontrado sem pulso. O PCC é um instrumento de autodeterminação do paciente, que contém de forma concisa os desejos ou limitação de terapia, endereços e números de telefone de familiares, de serviços e pessoas envolvidas no tratamento do paciente. Trata-se de um documento objetivo e que auxilia na tomada de decisão terapêutica em situações de emergência paliativa (Taghavi et al., 2012).

O Formulário de Diretivas Antecipadas (DA) padronizado, de uma página, foi utilizado em uma instituição de longa permanência do Canadá, tendo sido preenchido pelo residente capaz de tomar decisão ou pelo seu representante, caso não houvesse capacidade. Ele era mantido no prontuário para acesso rápido em caso de emergência e era composto por quatro categorias: tratamento ativo com transferência para o hospital e ressuscitação completa; tratamento ativo com transferência para o hospital, mas sem ressuscitação; tratamento médico ativo no local na casa de repouso; e medidas de conforto no local. Tais categorias foram agrupadas em duas únicas, a citar: aqueles que solicitaram transferência para o hospital ("sim, transferência para o hospital" - Yes, transfer to hospital - YTTH) e aqueles que não solicitaram transferência para o hospital (NTTH) (Nemiroff et al., 2019).

\section{Desafios da atuação profissional no atendimento pré-hospitalar em final de vida por doença avançada}

Nesta unidade, discorre-se sobre os desafios que os profissionais dos serviços de atendimento pré-hospitalar enfrentam no cotidiano. Desafios relacionados às condutas com pacientes e famílias, bem como desafios relacionados à organização e comunicação entre os serviços de saúde. Com base neles, são apresentadas as estratégias desenvolvidas pelos profissionais com vistas a amenizá-los.

Dentre os estudos que tiveram profissionais de saúde como participantes da pesquisa, o sexo masculino foi predominante, a idade média dos participantes ficou em torno de 38 anos (Ferrand et al., 2006; Jouffroy et al., 2017a; MurphyJones \& Timmons, 2016; Patterson et al., 2019; Stone et al., 2009; Taghavi et al., 2012), e médicos de APH ou atuantes em cuidados paliativos foram mais abordados (Ferrand et al., 2006; Jouffroy et al., 2017a; Waldrop et al., 2014, 2020; Wiese et al., 
2013). Os profissionais tinham experiência em serviço médico de emergência, variando entre dois e 20 anos (Clemency et al., 2019; Murphy-Jones \& Timmons, 2016; Stone et al., 2009).

Os desafios que os profissionais de saúde enfrentam durante sua atuação profissional, conforme alguns estudos demonstraram, versaram desde a falta de rastreabilidade das informações sobre o histórico de saúde do paciente, em razão da comunicação ineficiente entre os serviços de saúde (Jabre et al., 2008; Murphy-Jones \& Timmons, 2016), o conhecimento e treinamento insuficiente em cuidados paliativos e cuidados a pacientes em final de vida (Waldrop et al., 2014) até a falta de documentos de manifestação dos desejos do paciente (diretivas antecipadas) (Taghavi et al., 2012) ou, na sua existência, ausência de respaldo ético, além da exigência de realizar o atendimento de forma rápida (Davey et al., 2016).

Em um dos estudos foram identificadas nove categorias de desafios na atuação profissional: condições estruturais de atendimento de emergência pré-hospitalar, documentação e pedidos médicos, encontrar terapia centrada no paciente ideal, incerteza sobre as consequências legais, desafios no nível individual, desafios no nível da equipe, emoções do cuidador familiar, enfrentamento e compreensão da doença do paciente, desejos do paciente, enfrentamento e compreensão da doença do histórico social, cultural e religioso dos pacientes e familiares (Kamphausen et al., 2019).

Quanto às condutas dos profissionais do serviço de atendimento pré-hospitalar relacionadas ao próprio serviço, evidenciou-se dificuldade em distinguir um paciente doente que precisa de transferência urgente para o hospital de um paciente moribundo, que pode ser cuidado na comunidade até o momento de sua morte (Hoare et al., 2018), falta de compreensão sobre diretivas antecipadas de vontade e medo por não haver respaldo jurídico quanto a certas decisões, como, por exemplo, não iniciar reanimação cardiopulmonar (Stone et al., 2009). Ainda, identificou-se falta de conhecimento e receio legal quanto à prescrição de medicamentos opioides em altas doses (Kamphausen et al., 2019).

No que tange às condutas relacionadas à comunicação com outros serviços, identificou-se ausência ou dificuldade de acesso às informações do paciente em final de vida em um registro único, resistência em contatar o médico de família (Burnod et al., 2012) ou indisponibilidade desses quando contatados durante a madrugada ou finais de semana (Hoare et al., 2018).

Nessa direção, estudos apontaram que os profissionais do serviço de atendimento pré-hospitalar, geralmente, iniciam a ressuscitação em pacientes sem sinais vitais (Guru et al., 1999; Taghavi et al., 2012). Devido às regulamentações legais de emergência, embora possa existir uma diretiva antecipada recusando medidas de reanimação, elas são iniciadas e transfere-se os pacientes ao hospital (Guru et al., 1999; Stone et al., 2009; Wiese, et al., 2009).

Em algumas situações, a escolha de transportar um paciente em final de vida para o pronto socorro tem como finalidade estabilizar uma crise da família que se encontra desesperada frente à morte (Waldrop et al., 2015). Quando os pacientes não apresentam capacidade para tomar decisão, profissionais têm propensão a agir conforme o que consideram melhor em suas concepções, ponderando: diagnóstico, comorbidades, qualidade de vida, desejos conhecidos e condições atuais (Murphy-Jones \& Timmons, 2016).

Os achados indicam que o tempo médio de sobrevida após a reanimação é de três horas e nenhum paciente recebe alta hospitalar após a reanimação (Guru et al., 1999). Diante desses impasses, constatou-se que profissionais com mais anos de experiência possuem menor dificuldade de limitar, suspender ou não iniciar tratamentos, especialmente se há um registro das vontades (Taghavi et al., 2012; Wiese, et al., 2009).

\section{Atendimento pré-hospitalar e possíveis estratégias de cuidado diante do final da vida}

Como estratégias de cuidado diante do final da vida nos serviços de atendimento pré-hospitalar, identificou-se a introdução de um formulário de cuidados paliativos por escrito nos serviços médicos de emergência e educação jurídica correspondente sobre as diretivas antecipadas, como forma de reduzir as incertezas dos profissionais (Taghavi et al., 2012; Wiese et al., 2011). 
Os estudos demonstraram que profissionais com experiência ou formação adicional em cuidados paliativos sentiam-se mais seguros ao atender pacientes em final de vida (Feder et al., 2006; Wiese, et al., 2009). Dessa forma, uma das estratégias identificadas foi a integração dos temas cuidados paliativos e cuidados em final de vida em currículos da formação de profissionais de nível superior, técnico e também de cuidadores que atuam em instituições de longa permanência ou clínicas especializadas (Wiese et al., 2010). ssa educação pode ser realizada especialmente por meio de seminários ou com base na aprendizagem baseada em problemas. Deve abordar não somente diretrizes de cuidados (Clemency et al., 2019), como também estimular habilidades de resolução de conflitos e o gerenciamento das emoções da família e dos próprios profissionais (Waldrop et al., 2014), além de questões jurídicas em torno da validade das manifestações de vontade dos pacientes (Wiese et al., 2010).

Também foi enaltecida a importância do apoio das equipes de cuidados paliativos e integração entre os serviços da rede (Carron et al., 2014). A assistência de uma equipe profissional extra-hospitalar de cuidados paliativos (PCT) como suporte no acompanhamento médico e psicossocial foi uma possibilidade levantada e se mostrou eficiente (Wiese et al., 2009), reduzindo o número de chamadas aos serviços de urgência e emergência por pacientes e seus familiares e hospitalizações desnecessárias no final da vida (Wiese et al., 2008). Igualmente, mostrou-se pertinente a cooperação precoce entre uma equipe médica de emergência paliativa e equipes de atendimento pré-hospitalar (Wiese et al., 2013).

Integrar os cuidados paliativos no serviço médico de emergência pré-hospitalar requer educação formal e treinamento sobre aspectos éticos e práticos dos planos de cuidados avançados, estratégias de cuidados paliativos, tratamento da dor, cuidados com o paciente que está morrendo, notificação e anúncio do óbito à família, aspectos religiosos da morte e morrer e recusar intervenções que prolongam a vida (Carron et al., 2014).

Outro tipo de estratégia identificada foram formulários, instrumentos e programas que facilitem a assistência e o entendimento dos profissionais dos serviços de atendimento pré-hospitalar frente às situações de final de vida, fornecendo maiores informações sobre os pacientes, bem como maior respaldo para atuação.

Com o desenvolvimento do prontuário eletrônico e o aumento na velocidade da comunicação - através da internet -, é possível acessá-lo no ambiente extra-hospitalar, recomendando-se a inserção de diretivas antecipadas nesse espaço, para conhecimento de todos aqueles que assistem ao paciente ou os que virão a assistir (Jouffroy et al., 2017b). Compartilhar, em sistemas eletrônicos, os documentos e planos de cuidados - incluindo prescrições antecipadas para situações específicas propostos por equipes de consultoria que acompanham o paciente, pode melhorar o atendimento no final da vida e a qualidade da tomada de decisão (Patterson et al., 2019).

De igual modo, a obtenção de informações por meio de telefonema ao profissional de referência do paciente ou a equipes especializadas de cuidados paliativos, as quais podem atuar em regime de plantão, pode ser uma valiosa contribuição para a prática da Medicina de Urgência Pré-Hospitalar (Jouffroy et al., 2017a). Por fim, o desenvolvimento e aprimoramento da estrutura do Serviço Especializado de Cuidados Paliativos e a ampliação dos cuidados paliativos em nível ambulatorial se mostraram uma forma mais efetiva de reduzir o atendimento médico emergencial aos pacientes em final de vida e suas famílias (Wiese et al., 2013).

\section{Discussão}

Em relação ao perfil dos profissionais, nos artigos que tiveram essa população como participantes da pesquisa, foi identificada predominância do sexo masculino em comparação ao feminino. Também vale destacar o número expressivo da participação masculina na autoria dos artigos. Tal achado vai ao encontro da revisão integrativa, que avaliou o perfil, as dificuldades e as particularidades no trabalho de profissionais dos serviços de atendimento pré-hospitalar móvel e concluiu que a maioria dos profissionais eram do sexo masculino (Sousa et al., 2020). 
Entretanto, este resultado diverge do estudo que identificou o perfil das atividades de enfermeiros do SAMU de um estado da região Sul do Brasil (Luchtemberg \& Pires, 2016). Nele, 69,8\% dos participantes (de um total de 63) eram do sexo feminino. Um dado relacionado à característica histórica da enfermagem em ser composta majoritariamente por mulheres. Também diverge do perfil encontrado entre profissionais que atuam em equipes de cuidados paliativos, nas quais há predominância de mulheres, tanto em se tratando de profissionais de enfermagem quanto profissionais da medicina (Menezes \& Heilborn, 2007).

Com a emergência dos cuidados paliativos e sua instituição enquanto um saber que deve nortear as condutas na gestão sobre o final da vida e a morte, identifica-se o atravessamento de questões de gênero no reconhecimento que tais cuidados tiveram no contexto da área da saúde. Verifica-se hierarquização entre especialidades e as atribuições sociais sobre quem deve cuidar diante do nascimento e da morte (Menezes \& Heilborn, 2007). Nesse sentido, os cuidados com o corpo, a higiene, além de habilidades, como a comunicação, empatia, paciência e sensibilidade, são consideradas culturalmente como atributo das mulheres, cabendo a essas a responsabilidade pela implementação desse jeito específico de cuidar. Aos homens culturalmente se atribui características como objetividade e racionalidade, as quais convergem com os aspectos relacionados às tecnologias, cabendo a eles as áreas como a cirúrgica, a urgência e emergência, dentre outras. Denota-se, assim, que a humanização do processo de morrer e da morte caberia às mulheres, um pensamento que corrobora com a continuidade das dicotomias entre tecnologia e humanização, masculino e feminino (Menezes \& Heilborn, 2007).

Quanto aos países que mais publicaram sobre a temática, Estados Unidos da América (EUA), Alemanha e França se destacaram. Sendo que uma relação possível está no nível de desenvolvimento de cuidados paliativos nesses países. Segundo pesquisa que avaliou a qualidade de morte e do final da vida de 80 países, os Estados Unidos da América ficaram na $9^{\mathrm{a}}$ posição, a Alemanha em $7^{\circ}$ e a França em $10^{\circ}$ (The Economist, 2015). Os pesquisadores utilizaram como critérios: a presença de políticas públicas e serviços de cuidados paliativos, a qualidade do acesso da população a tais serviços, a oferta e condições de acesso aos opioides e, também, estratégias de difusão dos cuidados paliativos e dos documentos de manifestação de vontade, como as diretivas antecipadas na sociedade.

Além disso, um estudo identificou que maior nível de desenvolvimento de Cuidados Paliativos pode ser encontrado em países com baixos níveis de corrupção política, mortalidade infantil e mortes por doenças infecciosas, por exemplo (Clark et al., 2019). Nota-se, assim, que essa constatação também vai ao encontro dos países que mais publicaram estudos sobre atenção pré-hospitalar e cuidados paliativos. EUA, Alemanha e França apresentam altos níveis de desenvolvimento econômico, político e social, além de terem experienciado o processo de envelhecimento populacional desde a segunda metade do século XX, o que exigiu investimento em estratégias de atenção e cuidado à população idosa (Calha, 2015).

No que se refere às instituições de longa permanência de idosos (ILPI) e clínicas, o medo diante da fase agônica ou fase ativa de morte levou os profissionais a acionar os serviços de atendimento pré-hospitalar. Nesse sentido, pesquisa realizada com 20 profissionais da saúde atuantes em ILPIs, com o objetivo de conhecer suas vivências diante do processo de morrer e da morte, identificou que a impotência e a tristeza vivenciadas pelos profissionais possuem relação com a percepção da própria finitude (Oliveira et al., 2013).

Conforme constatado no presente estudo, muitas famílias recorrem aos serviços de atendimento pré-hospitalar por se sentirem inseguras diante de sintomas que se exacerbam no final da vida, como a dor e a dispneia (Burnod et al., 2012; Montgomery et al., 2017; Swetenham et al., 2014; Waldrop et al., 2020; Wiese et al., 2008). Por isso, acredita-se que o apoio por meio da educação junto a esses familiares, bem como a disponibilidade de suporte por telefone ou aplicativo de celular, pode auxiliar na redução de encaminhamentos desnecessários dos pacientes oncológicos em fase avançada aos serviços de urgência e emergência. Além disso, pode aliviar a sensação de desamparo das famílias. 
partir dos resultados desta revisão, percebeu-se que embora os pacientes desejassem permanecer em casa ou não desejassem ser transferidos para o hospital, houve dificuldades das famílias e dos profissionais do serviço de atendimento préhospitalar em respeitar essa vontade. O domicílio, nas últimas décadas, se tornou um cenário possível de atuação dos profissionais de saúde no final da vida, os quais adentram esse espaço com o intuito de propor cuidados, auxiliar as famílias e fornecer informações sobre comportamentos (Cordeiro, Oliveira, \& Kruse, 2020). No Brasil, uma forma de apoio para as famílias que desejam manter os pacientes em casa ocorre pelos serviços de atenção domiciliar (SAD), que permitem a desospitalização precoce e a continuidade do cuidado no domicílio, com amparo técnico, estrutural e emocional de equipes de saúde (Procópio et al., 2019).

Nessa direção, estudos internacionais têm destacado o domicílio como sendo o local de escolha das pessoas para finalizarem seus dias (Henriques et al., 2016; Loh et al., 2016), seguido por instituições de longa permanência (Loh et al., 2016). No cenário português, uma pesquisa evidenciou que, majoritariamente, o local de preferência em relação ao término dos dias está documentado no conteúdo disposto nas diretivas antecipadas de vontade. De maneira geral, os doentes optam por morrer em casa, mas barreiras como o medo de não ter suporte adequado e não saber lidar com possíveis intercorrências com o agravo dos sintomas impedem que isso se materialize (Henriques et al., 2016).

Esses apontamentos alertam para os motivos da resistência entre os profissionais de saúde para manter pacientes em final de vida no domicílio. Neste estudo, evidenciou-se fragilidade em relação aos aspectos legais e jurídicos aos quais os profissionais dos serviços de atendimento pré-hospitalar se sentem submetidos (Guru et al., 1999; Stone et al., 2009; Taghavi et al., 2012). Sendo assim, surge a necessidade de encontrar possíveis formas de manifestações da vontade do paciente sobre os desejos em final de vida.

Nesta revisão, diferentes tipos de registro e formas de manifestar vontades foram identificados (Nemiroff et al., 2019; Taghavi et al., 2012; Waldrop et al., 2020). Tais registros sobre os desejos de cuidados ou tratamentos em final de vida são fundamentais no atendimento pré-hospitalar, pois é esse serviço que geralmente atende chamadas para prestar cuidados no domicílio quando pacientes apresentam, por exemplo, agudização de sintomas ou encontram-se na iminência da morte. Caso não haja documentação de desejos no momento do atendimento, as vontades dos pacientes tendem a não ser respeitadas.

Um tipo de diretiva antecipada que se destacou neste estudo foi a ordem de não tentar ressuscitar (DNAR). Sobre a DNAR, recomenda-se que os adultos sejam abordados precocemente quanto às informações sobre o tempo de vida, o prognóstico da doença e os sintomas dela decorrentes e as consequências da ressuscitação para, assim, poderem manifestar seus desejos. Dentre as barreiras para maior realização desse tipo de diretiva estão as preocupações das famílias, a incerteza da pessoa sobre futuras mudanças no estado de saúde e a falta de habilidade dos profissionais (Fan et al., 2018).

Levando em conta os cenários culturais e de organização das políticas públicas de saúde, cada país aborda de forma diferente aspectos relacionados às DAV. No Brasil, não há legislação específica que contemple as DAV, mas existe uma resolução do Conselho Federal de Medicina (Resolução CFM 1.995/2012) que normatiza o documento no país. Também há, no momento atual, a tramitação no Senado Federal do Projeto de Lei no 149 de 2018, de autoria do senador Lasier Martins, que trata-se de uma primeira possibilidade de Lei acerca das DAV (Dadalto, 2016; Moscoso et al., 2021).

Em Porto Rico, primeiro país da América Latina a regulamentar as diretivas em 2001, a lei permite que cidadãos maiores de 21 anos possam manifestar-se sobre tratamentos e demais cuidados a serem prestados. Os trâmites também podem ser feitos por meio de procurador designado. Na Argentina, desde o ano de 2009 existe uma lei federal vigente que regulamenta as DAV. As diretivas podem ser revogadas pelo paciente a qualquer momento e só não são aceitas no país em casos de práticas que envolvam a eutanásia. No México, as diretivas podem incluir aspectos referentes à transplantes de órgãos e tecidos. No Uruguai, desde 2009, maiores de 18 anos com doença avançada, irreversível e incurável podem antecipar 
questões voltadas ao recebimento de intervenções e cuidados. Em todos os países citados, as DAV devem ser registradas em cartório para fins legais (Monteiro \& Silva, 2019).

Na Europa, desde 2012, em Portugal, há uma lei que regulamenta as DAV no país. As diretivas são feitas por meio de preenchimento de um formulário já disponível com o intuito de facilitar os trâmites, podendo a pessoa fazer ou não o uso desse documento. Ainda, faz-se necessário que cada pessoa ou procurador atualize as DAV a cada cinco anos. Na França, apesar do país dispor de legislação desde 2005, foi no ano de 2016 que houve a inclusão da obrigatoriedade de os médicos respeitarem as DAV na lei que dispõe sobre os direitos da pessoa em final de vida. As diretivas estão disponíveis para pessoas saudáveis e também para pessoas em situação de final de vida, podendo ser revogadas ou alteradas pelo paciente a qualquer momento (Dadalto, 2016).

A partir das análises, observou-se que, embora em alguns países as diretivas estejam contempladas em legislação, na maioria das vezes elas são desconhecidas ou não respeitadas, demonstrando o receio existente entre profissionais, pacientes, familiares e cuidadores. Ao encontro, uma pesquisa brasileira identificou desconhecimento, pouca ou nenhuma abordagem sobre temas relacionados ao final de vida durante a formação profissional, destacando a importância de ampliar as discussões e a divulgação sobre cuidados paliativos e documentos legais de manifestação de vontades, como o testamento vital (Chehuen Neto et al., 2015).

No Brasil, mesmo com a difusão sobre o conceito das DAV, profissionais manifestam insegurança, principalmente, pelo fato de não haver lei que ofereça amparo legal para o atendimento das DAV e por sentirem-se enfrentando um dilema ético (Saioron et al., 2017).

Outro tema emergente foi o desafio durante a atuação profissional nos serviços de atendimento pré-hospitalar. Destacam-se as limitações no adequado manejo dos sintomas, especialmente a dor e a dispneia (Waldrop et al., 2014). Frente a eles, os profissionais demonstraram receio em relação ao uso de opioides (Kamphausen et al., 2019).

Em cuidados paliativos, os opioides são utilizados para o tratamento da dor forte e também da dispneia. Tanto pacientes quanto profissionais de saúde têm medo em relação ao seu uso devido aos efeitos adversos, como: náusea, sonolência, constipação e depressão respiratória. O medo exacerbado desses medicamentos, por aspectos clínicos ou legais, pode gerar a "opiofobia", um problema mundial no que diz respeito ao uso, resultando em baixos índices de sua prescrição pelos profissionais de saúde (Calônego, 2020).

Outros desafios foram a comunicação entre os serviços de saúde, a falta de conhecimento e capacitação em cuidados paliativos, a falta de rastreabilidade de informações sobre o histórico de saúde do paciente e problemas estruturais das equipes de atendimento pré-hospitalar (Burnod et al., 2012; Hoare et al., 2018; Jabre et al., 2008; Murphy-Jones \& Timmons, 2016).

A ausência de educação permanente e a formação centrada nos aspectos técnicos do atendimento limitam a promoção do cuidado centrado no conforto ao final da vida (Carron et al., 2014). Os problemas estruturais dos sistemas de informação e comunicação nas centrais de regulação refletem no atendimento de menor qualidade, além da carência de profissionais para o posto médico na regulação e ambulâncias de suporte avançado de vida (O’Dwyer et al., 2017). Transportando-se ao cenário brasileiro, acredita-se que a melhor comunicação entre os serviços da atenção primária em saúde e os serviços de urgência e emergência, incluindo os serviços de atendimento pré-hospitalar, poderiam resultar em cuidados de final de vida mais efetivos no domicílio.

Assim, especificamente em relação aos cuidados paliativos, desde 2018, a Resolução no 41, de 31 de outubro de 2018, do Ministério da Saúde, estabelece diretrizes para a organização dos cuidados paliativos no Sistema Único de Saúde. Nesse documento, sugere-se a comunicação entre os serviços que compõem a Rede de Atenção em Saúde (RAS) e prioriza-se o espaço do domicílio como cenário de cuidado (Brasil, 2018). Os serviços de urgência e emergência devem assistir às pessoas 
visando o alívio de sintomas agudizados e a atenção hospitalar deve ser acionada quando não há manejo clínico em outros níveis de assistência (Brasil, 2018).

Dessa maneira, pensa-se que as estratégias de cuidado às pessoas em cuidados paliativos devem voltar-se ao domicílio, o que requer capacitação dos profissionais dos serviços de atendimento pré-hospitalar para o controle de sintomas, o apoio às famílias e para o não-encaminhamento desses pacientes aos hospitais.

Uma forma de prestar apoio aos profissionais dos serviços de atendimento pré-hospitalar pode se dar pela consultoria com equipes especializadas em cuidados paliativos. Essa foi uma experiência exitosa constatada em um dos estudos analisados (Patterson et al., 2019). No Brasil, esse apoio poderia ser viabilizado através das equipes de interconsultas, as quais já existem em alguns hospitais. Essas possuem experiência clínica no manejo de sintomas, já atuam nos hospitais orientando o tratamento, mas não necessariamente prestando os cuidados (Silva et al., 2020).

Outra forma de contribuir com os profissionais, diminuindo a falta de rastreabilidade das informações, conforme proposta dos estudos e pensando na realidade brasileira, seria a utilização de prontuários eletrônicos e com amplo acesso aos envolvidos na assistência. Ressalta-se que, em 2013, no Brasil, foi instituído o sistema e-SUS Atenção Básica (e-SUS AB), com a proposta de informatizar, qualificar, unificar e viabilizar as informações coletadas na atenção primária a partir do Sistema com Prontuário Eletrônico do Cidadão (PEC) (Conselho Nacional de Secretários de Saúde, 2013).

Acredita-se que a implantação do e-SUS AB representa um avanço para o Brasil no que concerne à qualificação e uso de informações registradas, uma das grandes fragilidades internacionais encontradas neste estudo. O Brasil pode explorar essa ferramenta, tornando as informações acessíveis aos serviços de saúde, desde os dados clínicos até os documentos de manifestação de vontade antecipada, facilitando, inclusive, o trabalho dos profissionais de atendimento pré-hospitalar.

Como limitações desta revisão, destacam-se o número limitado de bases de dados consultadas, a restrição de idiomas e o uso de descritores controlados. Também foram incluídos somente artigos disponíveis online e de forma gratuita ou com acesso pelo portal de periódicos da Coordenação de Aperfeiçoamento de Pessoal de Nível Superior (CAPES). Tais estratégias limitam o resultado de trabalhos possíveis.

\section{Conclusão}

Este estudo permitiu identificar as estratégias desenvolvidas internacionalmente para a atuação dos profissionais da atenção pré-hospitalar diante de pacientes com doença avançada em final de vida e suas famílias. Por se tratar de uma temática recente na área dos cuidados paliativos e os estudos terem sido desenvolvidos, em sua maioria, em países com qualidade do processo de morrer, foi possível apreender elementos que podem contribuir com o desenvolvimento de diretrizes em relação aos cuidados paliativos nos serviços brasileiros de atendimento pré-hospitalar.

Constatou-se que o serviço de atendimento pré-hospitalar é acionado por familiares ou cuidadores formais de pessoas com doença avançada em fim de vida no domicílio, em instituições de longa permanência ou clínicas, devido ao medo e à incapacidade de lidar com a exacerbação de sinais e sintomas inerentes à fase ativa de morte. Ao chegarem nos domicílios ou instituições, profissionais dos serviços de atendimento pré-hospitalar se sentem receosos e inseguros. Tais inseguranças estavam atreladas ao desconhecimento dos desejos dos pacientes, da cultura e crenças da família, dos cuidados terminais, dos cuidados paliativos e das formas de manejar sinais e sintomas. Outrossim, insegurança relacionada à necessidade de realizar manobras de suporte básico e avançado de vida, mesmo diante de quadros clínicos irreversíveis, sob risco de penalização ética e jurídica.

Como estratégias exitosas, identificou-se: existência, em alguns países, de manifestações antecipadas de desejos, com acesso aos profissionais do APH; prontuário eletrônico compartilhado, o que torna acessível o conhecimento sobre a história de saúde por todos os que assistem ao paciente, independentemente do tipo de serviço; educação para o final da vida, tendo 
sido valorizado e recomendado pelos profissionais o uso de metodologias ativas; consultoria com equipes especializadas em cuidados paliativos, o que auxilia os profissionais do APH na tomada de decisão. Considerando a estrutura dos serviços da rede de atenção em saúde no Brasil, acredita-se que há possibilidades de incorporar tais estratégias na organização das linhas de cuidado às pessoas com doença avançada no Sistema Único de Saúde.

Como contribuições, acredita-se que o estudo permitiu maior compreensão sobre as situações vivenciadas pelos profissionais do serviço de atendimento pré-hospitalar diante do final de vida por doença avançada. Como sugestão para trabalhos futuros, destaca-se a importância da realização de outras pesquisas, com delineamento qualitativo e com trabalho de campo, que possam identificar as limitações e potencialidades junto aos profissionais dos serviços de urgência e emergência, tanto em nível hospitalar quanto em nível pré-hospitalar, de modo a discutir, elaborar ou adaptar as estratégias identificadas com foco nas prioridades do cenário local.

\section{Referências}

Brasil. (2013). Manual instrutivo da Rede de Atenção às Urgências e Emergências no Sistema Único de Saúde (SUS). Ministério da Saúde. https://bvsms.saude.gov.br/bvs/publicacoes/manual_instrutivo_rede_atencao_urgencias.pdf

Brasil. (2003). Política Nacional de atenção às urgências. Ministério da Saúde. https://bvsms.saude.gov.br/bvs/publicacoes/politica_nac_urgencias.pdf

Brasil. (2018). Resolução $n^{o} 41$, de 31 de outubro de 2018. Ministério da Saúde.https://www.in.gov.br/materia/lasset_publisher/Kujrw0TZC2Mb/content/id/51520746/do1-2018-11-23-resolucao-n-41-de-31-de-outubro-de-2018-51520710

Burnod, A., Lenclud, G., Ricard-Hibon, A., Juvin, P., Mantz, J., \& Duchateau, F.-X. (2012). Collaboration between prehospital emergency medical teams and palliative care networks allows a better respect of a patient's will. European Journal of Emergency Medicine: Official Journal of the European Society for Emergency Medicine, 19(1), 46-47.

Calha, A. (2015). A condição sénior no Sul da Europa e na Escandinávia. Saúde e Sociedade, 24(2), 527-542.

Calônego, M. A. M. (2020). Dificuldades sociais, legais e burocráticas para prescrição de opioides [Tese de doutorado, Universidade Estadual Paulista "Júlio de Mesquita Filho"]. https://repositorio.unesp.br/handle/11449/192414

Carron, P.-N., Dami, F., Diawara, F., Hurst, S., \& Hugli, O. (2014). Palliative care and prehospital emergency medicine: Analysis of a case series. Medicine, 93(25), e128.

Carter, A. J. E., Arab, M., Harrison, M., Goldstein, J., Stewart, B., Lecours, M., Sullivan, J., Villard, C., Crowell, W., Houde, K., Jensen, J. L., Downer, K., \& Pereira, J. (2019). Paramedics providing palliative care at home: A mixed-methods exploration of patient and family satisfaction and paramedic comfort and confidence. CJEM, 21(4), 513-522.

Chehuen Neto, J. A., Ferreira, R. E., Silva, N. C. S. D., Delgado, Á. H. D. A., Tabet, C. G., Almeida, G. G., Vieira, I. F., Chehuen Neto, J. A., Ferreira, R. E., Silva, N. C. S. D., Delgado, Á. H. D. A., Tabet, C. G., Almeida, G. G., \& Vieira, I. F. (2015). Testamento vital: O que pensam profissionais de saúde? Revista Bioética, 23(3), 572-582.

Clark, J., Barnes, A., Campbell, M., \& Gardiner, C. (2019). A Life or “Good Death" Situation? A Worldwide Ecological Study of the National Contexts of Countries That Have and Have Not Implemented Palliative Care. Journal of Pain and Symptom Management, 57(4), 793-801.e11.

Clemency, B. M., Grimm, K. T., Lauer, S. L., Lynch, J. C., Pastwik, B. L., Lindstrom, H. A., Dailey, M. W., \& Waldrop, D. P. (2019). Transport Home and Terminal Extubation by Emergency Medical Services: An Example of Innovation in End-of-Life Care. Journal of Pain and Symptom Management, 58(2), $355-359$.

Cogo, S. B., Leon, P. B. D., Badke, M. R., Gomes, T. F., Malheiros, L. C. S., Ramos, T. K., Nietsche, E. A., Salbego, C., Sehnem, G. D., Lautenschleger, G., Rolim, I. L. T. P., Júnior, A. D., \& Silva, R. A. R. D. (2020). Palliative Care in the Emergency Room Scenario: Perceptions of Nurses and Physicians. Research \& Reviews: Journal of Nursing and Health Sciences, 6(2), 1-7.

Conselho Nacional de Secretários de Saúde. (2013). Estratégia e-SUS atenção básica e Sistema de informação em saúde da atenção básica (SISAB). Ministério da Saúde. https://www.conass.org.br/biblioteca/wp-content/uploads/2013/01/NT-07-2013-e-SUS-e-SISAB.pdf

Cordeiro, F. R., \& Kruse, M. H. L. (2019). It possible to die at home? Analysis of the brazilian and french scenarios. Texto e Contexto Enfermagem, 28, e20170602.

Cordeiro, F. R., Oliveira, S. G., Giudice, J. Z., Fernandes, V. P., \& Oliveira, A. T. (2020). Definitions for "palliative care", "end-of-life" and "terminally ill” in oncology: A scoping review. Enfermería: Cuidados Humanizados, 9(2), 205-228.

Cordeiro, F. R., Oliveira, S. G., \& Kruse, M. H. (2020). Cuidados ao final da vida no domicílio: Considerações sobre o Brasil e a França. Cultura de los cuidados, 24(57), 186-199.

Dadalto, L. (2016). A necessidade de um modelo de Diretivas Antecipadas de Vontade para o Brasil: Estudo comparativo dos modelos português e franceses. Revista M. Estudos sobre a morte, os mortos e o morrer, 1(2), 443-460. 
Davey, P. J., Lees, A. B., \& Godbold, R. (2016). Exploring New Zealand paramedic attitudes towards advance directives: an ethical analysis. Australasian Journal of Paramedicine, 13(4), 1-11.

Fan, S.-Y., Wang, Y.-W., \& Lin, I.-M. (2018). Allow natural death versus do-not-resuscitate: Titles, information contents, outcomes, and the considerations related to do-not-resuscitate decision. BMC Palliative Care, 17(1), 114.

Farinholt, P., Park, M., Guo, Y., Bruera, E., \& Hui, D. (2018). A Comparison of the Accuracy of Clinician Prediction of Survival Versus the Palliative Prognostic Index. Journal of Pain and Symptom Management, 55(3), 792-797.

Feder, S., Matheny, R. L., Loveless, R. S., \& Rea, T. D. (2006). Withholding resuscitation: A new approach to prehospital end-of-life decisions. Annals of Internal Medicine, 144(9), 634-640.

Ferrand, E., Marty, J., \& French LATASAMU Group. (2006). Prehospital withholding and withdrawal of life-sustaining treatments. The French LATASAMU survey. Intensive Care Medicine, 32(10), 1498-1505.

Ferrell, B. R., Temel, J. S., Temin, S., Alesi, E. R., Balboni, T. A., Basch, E. M., Firn, J. I., Paice, J. A., Peppercorn, J. M., Phillips, T., Stovall, E. L., Zimmermann, C., \& Smith, T. J. (2017). Integration of Palliative Care Into Standard Oncology Care: American Society of Clinical Oncology Clinical Practice Guideline Update. Journal of Clinical Oncology: Official Journal of the American Society of Clinical Oncology, 35(1), 96-112.

Formentin, M. S., Cordeiro, F. R., Zillmer, J. G. V., Oliveira, S. G., Zilli, F., \& Moscoso, C. R. (2021). Barreiras ao cuidado no final de vida em um serviço de urgência e emergência. Revista Uruguaya de Enfermería, 16(1), 1-13.

Gage, C. H., Geduld, H., \& Stassen, W. (2020). South African paramedic perspectives on prehospital palliative care. BMC Palliative Care, $19(1), 153$.

Gonsaga, R. A. T., Silva, E. M. da, Brugugnolli, I. D., Cabral, J. L., \& Thomé Neto, O. (2015). Padrão e necessidades de atendimento pré-hospitalar a idosos. Revista Brasileira de Geriatria e Gerontologia, 18, 19-28.

Guru, V., Verbeek, P. R., \& Morrison, L. J. (1999). Response of paramedics to terminally ill patients with cardiac arrest: An ethical dilemma. CMAJ: Canadian Medical Association Journal = Journal de l'Association Medicale Canadienne, 161(10), 1251-1254.

Henriques, J., Martins, L. S., \& Sapeta, P. (2016). Revisão sistemática da literatura: Preferência do local de cuidados dos doentes em fim de vida [Dissertação (Mestrado em Cuidados Paliativos), Instituto Politécnico $\quad$ de Castelo Branco]. https://repositorio.ipcb.pt/bitstream/10400.11/5407/1/Prefer\%C3\%AAncia\%20\%20Henriques_Martins.PDF.A.pdf

Hoare, S., Kelly, M. P., Prothero, L., \& Barclay, S. (2018). Ambulance staff and end-of-life hospital admissions: A qualitative interview study. Palliative Medicine, 32(9), 1465-1473.

Hui, D., Mori, M., Parsons, H. A., Kim, S. H., Li, Z., Damani, S., \& Bruera, E. (2012). The Lack of Standard Definitions in the Supportive and Palliative Oncology Literature. Journal of Pain and Symptom Management, 43(3), 582-592.

Hui, D., Nooruddin, Z., Didwaniya, N., Dev, R., De la Cruz, M., Kim, S. H., Kwon, J. H., Hutchins, R., Liem, C., \& Bruera, E. (2014). Concepts and Definitions for "Actively Dying," "End of Life," "Terminally Ill," "Terminal Care," and "Transition of Care": A Systematic review. Journal of Pain and Symptom Management, 47(1), 77-89.

International Association for Hospice and Palliative Care. (2013). What is Advanced kidney disease—Meaning and definition-Pallipedia. https://pallipedia.org/advanced-kidney-disease/

International Association for Hospice and Palliative Care. (2016). What is End-of-life care—Meaning and definition—Pallipedia. https://pallipedia.org/end-oflife-care/

International Association for Hospice and Palliative Care. (2021). What is Serious illness—Meaning and definition—Pallipedia. https://pallipedia.org/seriousillness/

Jabre, P., Combes, X., Marty, J., Margenet, A., \& Ferrand, E. (2008). The law number 2005-370 of April 22, 2005 concerning the patients' rights at the end of life: A case of prehospital medicine. Annales Francaises D'anesthesie Et De Reanimation, 27(11), 934-937.

Jouffroy, R., Michaloux, M., Khelifi, G., Guyard, A., Philippe, P., Carli, P., \& Vivien, B. (2017a). Retentissement psychologique des situations de fin de vie en médecine d'urgence préhospitalière chez les médecins du Samu de Paris (Samu 75). Journal Européen des Urgences et de Réanimation, 29(4), 288-295.

Jouffroy, R., Michaloux, M., Khelifi, G., Guyard, A., Philippe, P., Carli, P., \& Vivien, B. (2017b). Retentissement psychologique et connaissances par rapport à la fin de vie en médecine d'urgence pré-hospitalière chez les médecins du SMUR - résultats de l'enquête nationale de 2013. Journal Européen des Urgences et de Réanimation, 29(4), 296-302.

Kamphausen, A., Roese, H., Oechsle, K., Issleib, M., Zöllner, C., Bokemeyer, C., \& Ullrich, A. (2019). Challenges Faced by Prehospital Emergency Physicians Providing Emergency Care to Patients with Advanced Incurable Diseases. Emergency Medicine International, $2019,3456471$.

Kobewka, D., Ronksley, P., McIsaac, D., Mulpuru, S., \& Forster, A. (2017). Prevalence of symptoms at the end of life in an acute care hospital: A retrospective cohort study. CMAJ Open, 5(1), E222-E228.

Loh, A. Z. H., Tan, J. S. Y., Jinxuan, T., Lyn, T. Y., Krishna, L. K. R., \& Goh, C. R. (2016). Place of Care at End of Life: What Factors Are Associated With Patients' and Their Family Members' Preferences? American Journal of Hospice and Palliative Medicine ${ }^{\circledR}, 33(7), 669-677$.

Lord, B., Récoché, K., O’Connor, M., Yates, P., \& Service, M. (2012). Paramedics' perceptions of Their Role in Palliative Care: Analysis of Focus Group Transcripts. Journal of Palliative Care, 28(1), 36-40.

Luchtemberg, M. N., \& Pires, D. E. P. de. (2016). Nurses from the Mobile Emergency Service: Profile and developed activities. Revista Brasileira de Enfermagem, 69(2), 213-220. 
Maier, S. R. O., Ritter, M. F., Jorge, A. a. S., Agulh $\tilde{A}^{3}$, D. L. Z., Alves, E. V., Boff, T. R., Ferreira, A. F., Silva, G. A., Andrade, L. M. S., \& Silva, L. C. (2016). Glare of the notice of public policy in emergency from North of Mato Grosso: Integrative literature review. Scientific Electronic Archives, 9(2), 114119.

Matos, T. A. (2015). Cuidados paliativos: A realidade de uma unidade de emergência hospitalar e os caminhos na construção de diretrizes para o cuidado sob a ótica da enfermagem [Dissertação de Mestrado, Universidade Federal de Santa Catarina]. http://www.hu.ufsc.br/setores/enfermagem/wpcontent/uploads/sites/10/2014/10/2015-THAIS-ALVES-MATOS.pdf

Mendes, K. D. S., Silveira, R. C. de C. P., Galvão, C. M., Mendes, K. D. S., Silveira, R. C. de C. P., \& Galvão, C. M. (2019). Use of the bibliographic reference manager in the selection of primary studies in integrative reviews. Texto \&amp; Contexto - Enfermagem, 28, e20170204.

Menezes, R. A., \& Heilborn, M. L. (2007). A inflexão de gênero na construção de uma nova especialidade médica. Revista Estudos Feministas, 15(3), 563580.

Monteiro, R. da S. F., \& Silva, A. G. da. (2019). Diretivas antecipadas de vontade: Percurso histórico na América Latina. Revista Bioética, $27,86-97$.

Montgomery, C. L., Pooler, C., Arsenault, J. E., Berean, C., Sharman, R., Cameron, C. L., \& de Kock, I. (2017). Innovative Urgent Care for the Palliative Patient at Home. Home Healthcare Now, 35(4), 196-201.

Moscoso, C. R., Cordeiro, F. R., Marques, R. dos S., \& Silva, K. de O. (2021). Manifestação de dejesos ao final da vida. In F. R. Cordeiro, J. C. Fripp, \& S. G. Oliveira (Orgs.), Final de vida: Abordagem muldisciplinar ( $1^{\circ}$ ed, p. 99-108). Moriá.

Murphy-Jones, G., \& Timmons, S. (2016). Paramedics' experiences of end-of-life care decision making with regard to nursing home residents: An exploration of influential issues and factors. Emergency Medicine Journal: EMJ, 33(10), 722-726.

National Cancer Institute. (2011). Definition of advanced cancer-NCI Dictionary of Cancer Terms. https://www.cancer.gov/publications/dictionaries/cancerterms/def/advanced-cancer

Nemiroff, L., Marshall, E. G., Jensen, J. L., Clarke, B., \& Andrew, M. K. (2019). Adherence to "no transfer to hospital" advance directives among nursing home residents. Journal of the American Medical Directors Association, 20(11), 1373-1381.

O’Dwyer, G., Konder, M. T., Reciputti, L. P., Macedo, C., \& Lopes, M. G. M. (2017). O processo de implantação do Serviço de Atendimento Móvel de Urgência no Brasil: Estratégias de ação e dimensões estruturais. Cadernos de Saúde Pública, 33(7).

O’Dwyer, G., Machado, C. V., Alves, R. P., \& Salvador, F. G. (2016). Atenção pré-hospitalar móvel às urgências: Análise de implantação no estado do Rio de Janeiro, Brasil. Ciência \& Saúde Coletiva, 21, 2189-2200.

Oliveira, P. P. de, Amaral, J. G., Viegas, S. M. da F., \& Rodrigues, A. B. (2013). Percepção dos profissionais que atuam numa instituição de longa permanência para idosos sobre a morte e o morrer. Ciência \&amp; Saúde Coletiva, 18(9), 2635-2644.

Pastrana, T., De Lima, L., Sánchez-Cárdenas, M., Van Steijn, D., Garralda, E., Pons, J., \& Centeno, C. (2021). Atlas de Cuidados Paliativos en Latinoamérica 2020 ( $2^{\circ}$ ed). IAHPC Press.

Patterson, R., Standing, H., Lee, M., Dalkin, S., Lhussier, M., Exley, C., \& Brittain, K. (2019). Paramedic information needs in end-of-life care: A qualitative interview study exploring access to a shared electronic record as a potential solution. BMC Palliative Care, 18(1), 108.

Polit, D., \& Beck, C. T. (2019). Fundamentos de pesquisa em enfermagem: avaliação de evidências para a prática da enfermagem (9a ed). Artmed.

Procópio, L. C. R., Seixas, C. T., Avellar, R. S., Silva, K. L. da, Santos, M. L. de M. dos, Procópio, L. C. R., Seixas, C. T., Avellar, R. S., Silva, K. L. da, \& Santos, M. L. de M. dos. (2019). A Atenção Domiciliar no âmbito do Sistema Único de Saúde: desafios e potencialidades. Saúde em Debate, 43(121), 592604.

Radbruch, L., De Lima, L., Knaul, F., Wenk, R., Ali, Z., Bhatnaghar, S., Blanchard, C., Bruera, E., Buitrago, R., Burla, C., Callaway, M., Munyoro, E. C., Centeno, C., Cleary, J., Connor, S., Davaasuren, O., Downing, J., Foley, K., Goh, C., Pastrana, T. (2020). Redefining Palliative Care-A New Consensus-Based Definition. Journal of Pain and Symptom Management, 60(4), 754-764.

Rogers, I. R., Shearer, F. R., Rogers, J. R., Ross-Adjie, G., Monterosso, L., \& Finn, J. (2015). Paramedics' perceptions and educational needs with respect to palliative care. Australasian Journal of Paramedicine, 12(5), 1-8.

Saioron, I., Ramos, F. R. S., Amadigi, F. R., \& Diaz, P. da S. (2017). Diretivas antecipadas de vontade: Desafios legais e educacionais na visão de enfermeiros. Revista Eletrônica de Enfermagem, 19.

Santos, C. M. da C., Pimenta, C. A. de M., \& Nobre, M. R. C. (2007). A estratégia PICO para a construção da pergunta de pesquisa e busca de evidências. Revista Latino-Americana de Enfermagem, 15, 508-511.

Santos, A. F. J. dos, Ferreira, E. A. L., \& Guirro, Ú. B. do P. (2020). Atlas dos cuidados paliativos no Brasil 2019 (1ºd). Academia Nacional dos Cuidados Paliativos.

Silva, M. A. dos S., Diniz, M. A., Carvalho, R. T. de, Chiba, T., Mattos-Pimenta, C. A. de, Silva, M. A. dos S., Diniz, M. A., Carvalho, R. T. de, Chiba, T., \& Mattos-Pimenta, C. A. de. (2020). Equipe interconsultora em cuidados paliativos: alívio de sintomas nas primeiras 48 horas de hospitalização. Revista Brasileira de Enfermagem, 73(6), e20190391.

Sobral, M. A., Runa, D., \& Julião, M. (2019). Clinical signs of impending death: A retrospective descriptive analysis. Acta Médica Portuguesa, 32(12), 789789.

Sousa, B. V. N., Teles, J. F., Oliveira, E. F., Sousa, B. V. N., Teles, J. F., \& Oliveira, E. F. (2020). Perfil, dificuldades e particularidades no trabalho de profissionais dos serviços de atendimento pré-hospitalar móvel: Revisão integrativa. Enfermería Actual de Costa Rica, 38, 245-260. 
Stone, S. C., Abbott, J., McClung, C. D., Colwell, C. B., Eckstein, M., \& Lowenstein, S. R. (2009). Paramedic knowledge, attitudes, and training in end-of-life care. Prehospital and Disaster Medicine, 24(6), 529-534. https://doi.org/10.1017/s1049023x00007469

Swetenham, K., Grantham, H., \& Glaetzer, K. (2014). Breaking down the silos: collaboration delivering an efficient and effective response to palliative care emergencies. Progress in Palliative Care, 22(4), 212-218.

Taghavi, M., Simon, A., Kappus, S., Meyer, N., Lassen, C. L., Klier, T., Ruppert, D. B., Graf, B. M., Hanekop, G. G., \& Wiese, C. H. R. (2012). Paramedics experiences and expectations concerning advance directives: a prospective, questionnaire-based, bi-centre study. Palliative Medicine, 26(7), 908-916.

Tavares, A. P. dos S., Santos, C. G. da S., Tzanno-Martins, C., Barros Neto, J., Silva, A. M. M. da, Lotaif, L., Souza, J. V. L., Tavares, A. P. dos S., Santos, C. G. da S., Tzanno-Martins, C., Barros Neto, J., Silva, A. M. M. da, Lotaif, L., \& Souza, J. V. L. (2021). Cuidados de suporte renal: Uma atualização da situ ação atual dos cuidados paliativos em pacientes com DRC. Brazilian Journal of Nephrology, 43(1), 74-87.

The Economist. (2015). The quality of death: Ranking end-of-life care across the world (p. 71). Economist Intelligence Unit. http://www.eiu.comhttps://eiuperspectives.economist.com/sites/default/files/2015\%20EIU\%20Quality\%20of\%20Death\%20Index\%20Oct\%2029\%20FINAL.p dfsite_info.asp?info_name=qualityofdeath_lienfoundation\&page=noads

Waldrop, D., Clemency, B., Maguin, E., \& Lindstrom, H. (2015). Prehospital Providers' Perceptions of Emergency Calls Near Life's End. American Journal of Hospice and Palliative Medicine ${ }^{\circledR}, 32(2), 198-204$.

Waldrop, D. P., Clemency, B., Lindstrom, H. A., \& Clemency Cordes, C. (2015). "We Are Strangers Walking Into Their Life-Changing Event": how Prehospital Providers Manage Emergency Calls at the End of Life. Journal of Pain and Symptom Management, 50(3), 328-334.

Waldrop, D. P., Clemency, B., Maguin, E., \& Lindstrom, H. (2014). Preparation for frontline end-of-life care: exploring the perspectives of paramedics and emergency medical technicians. Journal of Palliative Medicine, 17(3), 338-341.

Waldrop, D. P., McGinley, J. M., \& Clemency, B. (2018). Mediating Systems of Care: Emergency Calls to Long-Term Care Facilities at Life's End. Journal of Palliative Medicine, 21(7), 987-991.

Waldrop, D. P., McGinley, J. M., Dailey, M. W., \& Clemency, B. (2019). Decision-Making in the Moments Before Death: Challenges in Prehospital Care. Prehospital Emergency Care: Official Journal of the National Association of EMS Physicians and the National Association of State EMS Directors, 23(3), $356-363$.

Waldrop, D. P., Waldrop, M. R., McGinley, J. M., Crowley, C. R., \& Clemency, B. (2020). Managing Death in the Field: Prehospital End-of-Life Care. Journal of Pain and Symptom Management, 60(4), 709-716.e2.

Wiese, C. H., Lassen, C. L., Bartels, U. E., Taghavi, M., Elhabash, S., Graf, B. M., \& Hanekop, G. G. (2013). International recommendations for outpatient palliative care and prehospital palliative emergencies - a prospective questionnaire-based investigation. BMC Palliative Care, 12(1), 10.

Wiese, C. H. R., Bartels, U. E., Marczynska, K., Ruppert, D., Graf, B. M., \& Hanekop, G. G. (2009). Quality of out-of-hospital palliative emergency care depends on the expertise of the emergency medical team-A prospective multi-centre analysis. Supportive Care in Cancer: Official Journal of the Multinational Association of Supportive Care in Cancer, 17(12), 1499-1506.

Wiese, C. H. R., Bartels, U. E., Ruppert, D. B., Graf, B. M., \& Hanekop, G. G. (2011). Prehospital emergency physicians' experiences with advance directives in Germany: A questionnaire-based multicenter study. Minerva Anestesiologica, 77(2), 172-179.

Wiese, C. H. R., Bartels, U. E., Ruppert, D., Marung, H., Luiz, T., Graf, B. M., \& Hanekop, G. G. (2009). Treatment of palliative care emergencies by prehospital emergency physicians in Germany: an interview based investigation. Palliative Medicine, 23(4), 369-373.

Wiese, C. H. R., Bartels, U. E., Zausig, Y. A., Pfirstinger, J., Graf, B. M., \& Hanekop, G. G. (2010). Prehospital emergency treatment of palliative care patients with cardiac arrest: A retrolective investigation. Supportive Care in Cancer: Official Journal of the Multinational Association of Supportive Care in Cancer, 18(10), 1287-1292.

Wiese, C. H., Vossen-Wellmann, A., Morgenthal, H. C., Popov, A. F., Graf, B. M., \& Hanekop, G. G. (2008). Emergency calls and need for emergency care in patients looked after by a palliative care team: retrospective interview study with bereaved relatives. BMC Palliative Care, 7(1), 11.

World Health Organization. (s/d). Advanced HIV disease (AHD). https://www.who.int/teams/global-hiv-hepatitis-and-stisprogrammes/hiv/treatment/advanced-hiv-disease\#: : :text=WHO\%20defines\%20advanced\%20HIV\%20disease,to\%20have\%20advanced\%20HIV\%20disease.

World Health Organization. (2018). Integrating palliative care and symptom relief into primary health care: A WHO guide for planners, implementers and managers. Genève: World Health Organization.

Worldwide Hospice Palliative Care Alliance. (2020). Global atlas of palliative care 2020. http://www.thewhpca.org/resources/global-atlas-on-end-of-life-care 